\title{
THE SHANNON-MCMILLAN-BREIMAN THEOREM BEYOND AMENABLE GROUPS
}

\author{
AMOS NEVO AND FELIX POGORZELSKI
}

\begin{abstract}
We introduce a new isomorphism-invariant notion of entropy for measure preserving actions of arbitrary countable groups on probability spaces, which we call orbital Rokhlin entropy. It employs Danilenko's orbital approach to entropy of a partition, and is motivated by Seward's recent generalization of Rokhlin entropy from amenable to general groups. A key ingredient in our approach is the use of an auxiliary probability-measure-preserving hyperfinite equivalence relation. Under the assumption of ergodicity of the auxiliary equivalence relation, our main result is a Shannon-McMillan-Breiman pointwise almost sure convergence theorem for the orbital entropy of partitions in measure-preserving group actions, the first such convergence result going beyond the realm of amenable groups. As a special case, we obtain a Shannon-McMillan-Breiman theorem for all strongly mixing actions of any countable group. Furthermore, we compare orbital Rokhlin entropy to Rokhlin entropy, and using an important recent result of Seward we show that they coincide for free, ergodic actions of any countable group.

Finally, we consider actions of non-Abelian free groups and demonstrate the geometric significance of the entropy equipartition property implied by the Shannon-McMillan-Breiman theorem. We show that the orbital entropy of a partition is the limit of the information functions of the sequence of partitions arising from refining any given finite partition along almost every horoball in the group.
\end{abstract}

\section{Contents}

1. Introduction and statement of main results

1.1. Shannon-McMillan-Breiman theorem for free groups

1.2. Historical background

1.3. Probability measure preserving actions of groups and cocycle extensions

1.4. Definition of orbital Rokhlin entropy

1.5. Orbital Rokhlin entropy, finitary entropy and Rokhlin entropy

1.6. The Shannon-McMillan-Breiman theorem

Acknowledgements.

2. Amenable equivalence relations

2.1. Measurable equivalence relations

2.2. Hyperfiniteness and amenability

3. Pointwise covering lemmas

4. Proof of the Shannon-McMillan-Breiman theorem

5. Amenable relations, injective cocycles and ergodic extensions

5.1. Groups admitting injective cocycles

5.2. Ergodicity of cocycle extensions

5.3. Ergodicity of the extended relation for strongly mixing actions

5.4. Ergodicity of the extended relation via weakly mixing Bernoulli actions

6. The free group

6.1. The boundary of the free group

6.2. The horospherical relation and the fundamental cocycle

Date: March 14, 2019

Key words and phrases. Ergodic group actions, entropy, measured equivalence relations, hyperfiniteness.

The first author acknowledges the support of ISF Moked grant \# 2095/15. The second author gratefully acknowledges support through a Technion post-doctoral Fine fellowship. 
6.3. Shannon-McMillan-Breiman theorem for the free groups

\section{INTRODUCTION AND STATEMENT OF MAIN RESUlTS}

1.1. Shannon-McMillan-Breiman theorem for free groups. The main result of this paper is the Shannon-McMillan-Breiman entropy equipartition theorem stated in Theorem 1.8 below. To explain our main result, let us begin by stating it in a special case, namely that of probabilitymeasure-preserving (p.m.p.) actions of the free group $\mathbb{F}_{r}, 2 \leq r<\infty$. We refer to section 6 for full discussion and proof. We recall that the Cayley graph of $\mathbb{F}_{r}$ with respect to free generators can be identified with a $2 r$-valent tree, where the group elements are identified with its vertices. We recall the associated word-metric balls $B_{n}(e)$ centered at the identity $e$, the boundary $\partial \mathbb{F}_{r}$ identified with the Cantor set of infinite reduced words, and the horoballs $B^{\eta}$ determined by a boundary point $\eta \in \partial \mathbb{F}_{2 r}$, passing at the identity $e$. The set $B_{2 n} \cap B^{\eta}:=B_{2 n}^{\eta}$ are called the horospherical balls determined by $\eta$, and they form a sequence of increasing finite sets, exhausting the horoball $B^{\eta}$. We can now state the following Shannon-McMillan-Breiman pointwise convergence theorem for actions of $\mathbb{F}_{r}$ (See Subsection 1.6 for all unexplained notions.)

Theorem 1.1. Let $(X, \mu)$ be an essentially free p.m.p. action of the free group $\mathbb{F}_{r}, 2 \leq r<\infty$. Assume that the action is ergodic under the index 2 subgroup of even words $\mathbb{F}_{r}^{e}$. Fix any finite partition $\mathcal{P}$ of $X$. For a boundary point $\eta \in \partial \mathbb{F}_{r}$, consider the sequence of partitions obtained from $\mathcal{P}$ by refining it along the horospherical balls $B_{2 k}^{\eta}$ determined by $\eta$, namely the partitions $\bigvee_{g \in B_{2 k}^{\eta}} g \mathcal{P}$. Then the normalized information functions of the refined partitions converge to a constant value $h_{\mathcal{P}}^{*}$, for almost every $x \in X$ and for almost every $\eta \in \mathbb{F}_{r}$.

The infimum of $h_{\mathcal{P}}^{*}$ over all countable generating partitions of $X$ is an isomorphism-invariant of the action denoted $h^{\mathrm{OR}}\left(\mathbb{F}_{r} \curvearrowright X\right)$, and will be called the orbital Rokhlin entropy. It coincides with the Rokhlin entropy and with the finitary entropy of the free group action.

1.2. Historical background. The concept of entropy for a measure-preserving transformation on a probability space was defined by Kolmogorov and Sinai, who introduced it as an isomorphism invariant for dynamical systems [Ko58, Ko59, Si59]. For a fixed finite partition of the probability space Breiman Br57] proved pointwise almost-everywhere convergence of the information function associated with the partition, thus establishing asymptotic "entropy equipartition" under the transformation. Incorporating previous results of Shannon Sh48 and McMillan Mc53, pointwise almost-everywhere entropy equipartition is usually referred to as the Shannon-McMillan-Breiman theorem.

The generalization of Kolmogorov-Sinai entropy to p.m.p. actions of countable amenable groups was developed by several authors, including Kieffer [Ki75, Ollagnier O185 and Ornstein-Weiss OW87. In particular, Ornstein-Weiss raised the natural question of extending the entropy equipartition theorem to actions of amenable groups, and proved an analog of the ShannonMcMillan-Breiman theorem along regular Følner sequences in amenable groups. For ergodic actions, this result was generalized by Lindenstrauss to tempered Følner sequences [Li01] (satisfying a very mild growth condition). The method of proof developed in Li01 motivate the techniques developed and used in the present paper. In the survey paper We03 Weiss gives a combinatorial proof in the case of possibly non-ergodic actions and for general tempered Følner sequences.

The development of entropy theory for p.m.p. actions of non-amenable groups has been a longstanding open problem, which has seen revolutionary developments in the past decade, initiated by the breakthrough work on sofic entropy by Bowen Bo10, Bo12, and its refinements by Kerr and Li KL11, KL13. In a separate very important recent development, for ergodic p.m.p. actions of general countable groups Seward introduced the notion of Rokhlin entropy Se14, Se15b. In Se16] Seward establishes a remarkable inequality which implies that Rokhlin entropy coincides with another notion of entropy, which we will call finitary entropy. Seward's inequality will play a crucial role in our considerations below. 
These developments naturally raise the question of the validity of the Shannon-McMillanBreiman theorem in the far more general context of actions of non-amenable groups. Motivated by an approach developled by Danilenko and Park for actions of amenable groups Da01, DP02, we define the orbital entropy for a partition, for any ergodic p.m.p. action of any countable group. With these values at hand, we define the of concept orbital Rokhlin entropy of the underlying group action. We will show using Seward's inequality that it fact coincides with Rokhlin entropy for free actions, cf. Proposition [1.7. Most importantly, for orbital entropy, we establish a general Shannon-McMillan-Breiman theorem, cf. Theorem 1.8.

Let us now turn to describe the general context and the main results.

1.3. Probability measure preserving actions of groups and cocycle extensions. Throughout the paper, we consider standard Borel equivalence relations with countable classes $\mathcal{R} \subset Y \times Y$, where $(Y, \nu)$ is a probability space. $[y]=\mathcal{R}(y) \subset Y$ denotes the $\mathcal{R}$-class of $y \in Y$. We assume that the equivalence relation preserves the probability measure $\nu$. It is further assumed that $\mathcal{R}$ is hyperfinite, or equivalently, that $\mathcal{R}$ is amenable in the sense of [CFW81. Thus $\mathcal{R}$ can be written as an increasing union of equivalence subrelations $\mathcal{R}_{n} \subset \mathcal{R}$, each with finite classes, i.e.

$$
\mathcal{R}(y):=\bigcup_{n=1}^{\infty} \mathcal{R}_{n}(y), \text { for } \nu \text {-almost every } y \in Y .
$$

Such a representation of $\mathcal{R}$ will be called a hyperfinite exhaustion. If for every $n$, the relations $\mathcal{R}_{n}$ are bounded, namely the size of the equivalence classes $\mathcal{R}_{n}(y)$ is essentially bounded (with a bound depending on $n$ ), we will call the representation a bounded hyperfinite exhaustion. Let us note that hyperfinite relations always admit a bounded hyperfinite exhaustion, as will be seen below. In fact it is possible to construct a hyperfinite exhaustion satisfying $\left|\mathcal{R}_{n}(y)\right| \leq n$ almost surely, as noted in We84 in a more general context.

Throughout the paper, $\Gamma$ denotes a countable group. The collection of all finite subsets of $\Gamma$ is denoted by $\operatorname{Fin}(\Gamma)$. We consider a probability measure preserving (p.m.p.) ergodic action $\Gamma \curvearrowright(X, \lambda)$, and aim to define a notion of entropy for the action.

To that end, assume that there is an amenable p.m.p. equivalence relation $\mathcal{R}$ over $(Y, \nu)$, admitting a measurable cocycle $\alpha: \mathcal{R} \rightarrow \Gamma$. Thus for $\nu$-almost every $y \in Y$ and every $w, u, z \in$ $[y]=\mathcal{R}(y)$, the cocycle identity holds :

$$
\alpha(z, u)=\alpha(z, w) \cdot \alpha(w, u) .
$$

A crucial construction in our discussion is the equivalence relation, denoted $\mathcal{R}^{X}$, which is the extension of the equivalence relation $\mathcal{R}$ by the cocycle $\alpha$ and the $\Gamma$-action on $X$. The extended equivalence relation $\mathcal{R}^{X}$ over $(X \times Y, \lambda \times \nu)$ is defined by the condition

$$
\left((x, y),\left(x^{\prime}, y^{\prime}\right)\right) \in \mathcal{R}^{X} \Longleftrightarrow y \mathcal{R} y^{\prime} \text { and } x=\alpha\left(y, y^{\prime}\right) x^{\prime} .
$$

Assuming that the measure $\nu$ is $\mathcal{R}$-invariant, it follows that $\lambda \times \nu$ is $\mathcal{R}^{X}$-invariant, since the $\Gamma$-action on $X$ preserves $\lambda$. The projection map $\pi: \mathcal{R}^{X} \rightarrow \mathcal{R}$ given by $(x, y) \rightarrow y$ is class injective, namely injective on almost every $\mathcal{R}^{X}$-equivalence class.

Further, it is well known that an extension of an amenable action is amenable, and thus in particular if $\mathcal{R}$ is amenable, so is $\mathcal{R}^{X}$. Thus, when $\mathcal{R}$ is hyperfinite, so is $\mathcal{R}^{X}$. But since the extension is class-injective, in fact every hyperfinite exhaustion $\left(\mathcal{R}_{n}\right)$ of $\mathcal{R}$ can be canonically lifted to a hyperfinite exhaustion $\left(\mathcal{R}_{n}^{X}\right)$ of $\mathcal{R}^{X}$, via $\mathcal{R}_{n}^{X}((x, y))=\left\{(\alpha(z, y) x, z) ; z \in \mathcal{R}_{n}(y)\right\}$. Note that if $\left(\mathcal{R}_{n}\right)$ is a bounded hyperfinite exhuastion, then so is $\left(\mathcal{R}_{n}^{X}\right)$, with the same bounds on the equivalence classes.

The cocycle $\alpha$ is called class injective if for $\nu$-almost every $y \in Y$, we have that $\alpha(y, z) \neq \alpha(y, w)$ whenever $w \neq z$. In order to avoid degenerate cases, we will assume in the sequel that the cocycle under consideration is class injective.

1.4. Definition of orbital Rokhlin entropy. In order to define the measure theoretic orbital entropy of the p.m.p. $\Gamma$-action on $X$, let us begin by recalling the following construction, due to 
Danilenko Da01]. First, for a countable measurable partition $\mathcal{P}=\left\{A_{i} ; i \in I\right\}$ of $X$ the Shannon entropy $H(\mathcal{P})$ is defined as

$$
H(\mathcal{P}):=-\sum_{A \in \mathcal{P}} \lambda(A) \log (\lambda(A))
$$

where we use the convention that $0 \cdot \log 0=0$. For two countable partitions $\mathcal{P}$ and $\mathcal{Q}$, their common refinement is $\mathcal{P} \vee \mathcal{Q}:=\{P \cap Q \mid P \in \mathcal{P}, Q \in \mathcal{Q}\}$. For a finite set $F \in F i n(\Gamma)$, we set

$$
\mathcal{P}^{F}:=\bigvee_{g \in F} g^{-1} \mathcal{P}
$$

where $g^{-1} \mathcal{P}=\left\{g^{-1} A_{i} ; i \in I\right\}$. If $F$ is the empty set, then we define $\mathcal{P}^{F}$ to be the trivial partition, which of course has Shannon entropy zero. Given two partitions $\mathcal{P}$ and $\mathcal{Q}, \mathcal{Q}$ is called finer than $\mathcal{P}$ or a refinement of $\mathcal{P}$, denoted $\mathcal{Q} \geq \mathcal{P}$, if for every $Q \in \mathcal{Q}$, there is some $P \in \mathcal{P}$ such that $Q \subseteq P$ up to $\lambda$-measure zero.

Now for a subset function $\mathcal{F}: Y \rightarrow \operatorname{Fin}(Y)$ (as always, with $\mathcal{F}(y) \subset \mathcal{R}(y)$ a.e.) and a countable partition $\mathcal{P}$ of $X$ with $H(\mathcal{P})<\infty$, we consider the entropy function, defined by Danilenko in Da01:

$$
h^{\mathcal{P}}(\mathcal{F}): Y \rightarrow[0, \infty): h^{\mathcal{P}}(\mathcal{F})(y):=H\left(\bigvee_{z \in \mathcal{F}(y)} \alpha(z, y)^{-1} \mathcal{P}\right)
$$

In was noted by Danilenko in Da01, Cor. 2.7] that one can attach a notion of orbital entropy to every partition $\mathcal{P}$ with finite Shannon entropy, and that the following holds.

Theorem 1.2 (see Da01, Cor. 2.7] and [DP02, Prop. 1.2]). Given a class injective cocycle on $\mathcal{R}$, for every countable partition $\mathcal{P}$ of $X$ with $H(\mathcal{P})<\infty$, there is a number $h_{\mathcal{P}}^{*}(\alpha)$ such that for every bounded hyperfinite exhaustion $\left(\mathcal{R}_{n}\right)$,

$$
h_{\mathcal{P}}^{*}(\alpha):=\lim _{n \rightarrow \infty} \int_{Y} \frac{h^{\mathcal{P}}\left(\mathcal{R}_{n}\right)(y)}{\left|\mathcal{R}_{n}(y)\right|} d \nu(y) .
$$

Let us highlight the crucial fact that it is part of the conclusion of Theorem 1.2 that the limit is independent of the choice of the bounded hyperfinite exhaustion.

Remark 1.3. As can be seen from the proof of Theorem 1.2 the assumption that the cocycle being class injective is not necessary for this convergence result. But in our discussion below we will dispense with this additional generality and focus on the set-up explained above of free p.m.p. actions of countable groups. The injectivity assumption then makes sure that the values $h_{\mathcal{P}}^{*}(\alpha)$ accurately reflect entropy theoretic information regarding the action of $\Gamma$ on $X$.

Furthermore, the results we develop below can be formulated more generally, for all classinjective extensiosn of arbitrary hyperfinite relations. But again, we will focus below on our main goal, which is the development of entropy theory for p.m.p. actions of non-amenable groups.

Following Da01, the number $h_{\mathcal{P}}^{*}(\alpha)$ shall be called the orbital entropy of the partition $\mathcal{P}$ for the action $\Gamma \curvearrowright(X, \lambda)$. Danilenko [Da01, Def. 2.4] proceeds to define the orbital entropy of the associated dynamical systems as the supremum of $h_{\mathcal{P}}^{*}(\alpha)$ over all finite partitions.

We propose a different definition, motivated by the developments in entropy theory for nonamenable groups due to Bowen and to Seward, particularly by Seward's recent work on Rokhlin entropy (see the discussion below). To define measure-theoretic entropy for the dynamical system $\Gamma \curvearrowright(X, \lambda)$ itself, we restrict ourselves to generating partitions, i.e. countable partitions $\mathcal{P}$ for $X$ such that (modulo null sets)

$$
\bigvee_{g \in \Gamma} g \mathcal{P}=\mathcal{B}
$$

Definition 1.4 (Orbital Rokhlin entropy). Let $(X, \lambda)$ be a p.m.p. ergodic action of $\Gamma$. Let $\alpha: \mathcal{R} \rightarrow \Gamma$ be a class injective cocycle defined on a hyperfinite relation. The number

$$
h^{\mathrm{OR}}(\Gamma \curvearrowright X):=\inf \left\{h_{\mathcal{P}}^{*}(\alpha) \mid \mathcal{P} \text { countable, generating partition }\right\}
$$


is called the orbital Rokhlin entropy for the action $\Gamma \curvearrowright(X, \lambda)$ and for the injective cocycle $\alpha: \mathcal{R} \rightarrow \Gamma$.

As we shall see presently, the orbital Rokhlin entropy of a free ergodic action is an intrinsic invariant, independent of the p.m.p. hyperfinite relation $\mathcal{R}$, the bounded hyperfinite exhaustion $\mathcal{R}_{n}$, and the class injective cocycle $\alpha$ used to define it. This remarkable fact is ultimately based on the important recent result established by Seward relating two completely different notions of entropy, which we now proceed to describe.

1.5. Orbital Rokhlin entropy, finitary entropy and Rokhlin entropy. The notion of Rokhlin entropy has its origin in Rokhlin's studies of entropy for a single transformation. It has recently been greatly generalized and studied intensively in the context of general group actions by Seward in the series of papers [Se14, Se15b, Se16]. In particular, Seward defines

Definition 1.5 (Rokhlin entropy). Let $\Gamma \curvearrowright(X, \lambda)$ be a p.m.p. ergodic group action. Then, the number

$$
h^{\mathrm{Rok}}(\Gamma \curvearrowright X):=\inf \{H(\mathcal{P}) \mid \mathcal{P} \text { countable, generating partition }\}
$$

is called the Rokhlin entropy of the group action.

Seward and Tucker-Drob showed in ST12 that for free ergodic actions of amenable groups, Rokhlin entropy coincides with the classical Kolmogorov-Sinai entropy. In a recent important breakthrough Seward Se16] established the following upper bound for the Rokhlin entropy for every countable, generating partition $\mathcal{P}$, assuming the $\Gamma$ action on $X$ is essentially free.

$$
h^{\mathrm{Rok}}(\Gamma \curvearrowright X) \leq \inf _{T \in \operatorname{Fin}(\Gamma)} \frac{1}{|T|} H\left(\bigvee_{g \in T} g^{-1} \mathcal{P}\right) .
$$

(In fact, Theorem 1.5 in Se16] shows a stronger statement involving entropy conditioned on $\Gamma$ invariant $\sigma$-algebras.)

Let us now note that by the standard subadditivity property of Shannon entropy, the right hand side of (1.1) is bounded from above by $H(\mathcal{P})$. Hence, by passing to the infimum over all generating partitions, we obtain in fact equality of the lower and the upper bound. Let us therefore define the following notion of finitary entropy.

Definition 1.6 (Finitary entropy).

$$
h^{\mathrm{fin}}(\Gamma \curvearrowright X):=\inf \left\{\inf _{T \in F i n(\Gamma)} H\left(\bigvee_{g \in T} g^{-1} \mathcal{P}\right) /|T| \mid \mathcal{P} \text { countable, generating partition }\right\} .
$$

By the preceding discussion, it follows from Seward's inequality (1.1) that these two notions of entropy coincide for ergodic (essentially) free actions: $h^{\text {fin }}(\Gamma \curvearrowright X)=h^{\mathrm{Rok}}(\Gamma \curvearrowright X)$.

Using this result, it is easy to see that our definition of orbital Rokhlin entropy above gives rise to the same value as well.

Proposition 1.7. Assume that $\Gamma \curvearrowright(X, \lambda)$ is an ergodic essentially free p.m.p. action. Then, for every amenable p.m.p. equivalence relation $\mathcal{R}$ over $(Y, \nu)$ and every class injective cocycle $\alpha: \mathcal{R} \rightarrow \Gamma$, we obtain

$$
h^{\mathrm{fin}}(\Gamma \curvearrowright X)=h^{\mathrm{OR}}(\Gamma \curvearrowright X, \alpha)=h^{\mathrm{Rok}}(\Gamma \curvearrowright X) .
$$

Proof. Let $\mathcal{P}$ be a countable partition with finite Shannon entropy. Assume that $\left(\mathcal{R}_{n}\right)$ is any bounded hyperfinite exhaustion for $\mathcal{R}$. Since the cocycle $\alpha$ is class injective, and $\mathcal{R}_{n}(y)$ is a finite set almost surely, we obtain

$$
\inf _{T \in \operatorname{Fin}(\Gamma)} \frac{1}{|T|} H\left(\bigvee_{g \in T} g^{-1} \mathcal{P}\right) \leq \frac{h^{\mathcal{P}}\left(\mathcal{R}_{n}\right)(y)}{\left|\mathcal{R}_{n}(y)\right|} \leq H(\mathcal{P})
$$

for each $n \in \mathbb{N}$ and for $\nu$-almost every $y \in Y$. Note that these inequalities remain valid even if $H(\mathcal{P})=\infty$. In the case that there exist generating partitions with finite Shannon entropy, we integrate over $Y$ and pass to the limit as $n \rightarrow \infty$. By Theorem 1.2 we conclude that $h^{\text {fin }}(\Gamma \curvearrowright$ $X) \leq h_{\mathcal{P}}^{*}(\alpha) \leq H(\mathcal{P})$ independently of the cocycle $\alpha$. Now taking the infimum over all generating 
partitions, since by the discussion above $h^{\operatorname{Rok}}(\Gamma \curvearrowright X)=h^{\mathrm{fin}}(\Gamma \curvearrowright X)$, the above inequality immediately implies equality of all three notions of entropy.

1.6. The Shannon-McMillan-Breiman theorem. The generalization that we propose of the classical Shannon-McMillan-Breiman theorem concerns pointwise almost everywhere convergence of a sequence of natural information functions on $X$, the space on which $\Gamma$ acts by measurepreserving transformations. For a given countable partition $\mathcal{P}$ of $X$ with $H(\mathcal{P})<\infty$, we set

$$
\mathcal{J}(\mathcal{P})(x):=-\log \lambda(\mathcal{P}(x)),
$$

where we define $\mathcal{P}(x)$ to be the unique set $A \in \mathcal{P}$ containing the point $x$ (namely the $\mathcal{P}$-name of $x$ ). Note that by definition, we have

$$
H(\mathcal{P})=\int_{X} \mathcal{J}(\mathcal{P})(x) d \lambda(x) .
$$

Given an ergodic p.m.p. group action $\Gamma \curvearrowright(X, \lambda)$, and a class-injective cocycle $\alpha: \mathcal{R} \rightarrow \Gamma$ consider the extended relation $\mathcal{R}^{X}$ over $X \times Y$. For the proof of the Shannon-McMillan-Breiman theorem, we will assume that the extended relation $\mathcal{R}^{X}$ is ergodic. Though being a non-trivial assumption on the equivalence relation and the action under consideration, ergodicity of the extension is satisfied in many situations. One important example is that of arbitrary ergodic actions of irreducible lattices in connected semisimple Lie groups with finite center. In general, a sufficient condition in order to guarantee ergodicity of $\mathcal{R}^{X}$ is weak mixing of the relation $\mathcal{R}$ over $(Y, \nu)$, as defined in BN13a. For a more detailed elaboration of these issues, we refer the reader to Section 7.

We are now able to state our main result, namely the Shannon-McMillan-Breiman pointwise convergence theorem for actions of non-amenable groups.

Theorem 1.8. Let $\Gamma \curvearrowright(X, \lambda)$ be an ergodic essentially free p.m.p. action. Assume that $\mathcal{R}$ is an amenable, p.m.p. equivalence relation, and $\alpha: \mathcal{R} \rightarrow \Gamma$ is a class injective cocycle, such that the extended relation $\mathcal{R}^{X}$ is ergodic. Then, for every bounded hyperfinite exhaustion $\left(\mathcal{R}_{n}\right)$ satisfying the growth condition

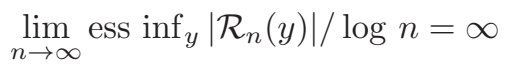

the information functions satisfy the following convergence property. Given a finite partition $\mathcal{P}$ of $X$, for $(\lambda \times \nu)$-almost every $(x, y) \in X \times Y$,

$$
\lim _{n \rightarrow \infty} \frac{\mathcal{J}\left(\mathcal{P}^{\mathcal{R}_{n}(y)}(x)\right)}{\left|\mathcal{R}_{n}(y)\right|}=h_{\mathcal{P}}^{*}(\alpha),
$$

where $h_{\mathcal{P}}^{*}(\alpha)$ is the orbital entropy of the partition $\mathcal{P}$.

Given any countable group $\Gamma$, a suitable equivalence relation $\mathcal{R}$ and cocycle $\alpha: \mathcal{R} \rightarrow \Gamma$ can be found, such that Theorem 1.8 applies to all strongly mixing action of $\Gamma$. We will discuss this matter in detail in $\$ 5.3$ below.

Let us note the following comments regarding the assumptions of Theorem 1.8

1) the growth condition on the $\left(\mathcal{F}_{n}\right)$ is very mild, and in fact one can always find bounded hyperfinite exhaustions which satisfy it. To see this, recall that any two ergodic p.m.p. actions of amenable groups are orbit equivalent. This fact goes back to Dye Dy59 for a pair of (ergodic) $\mathbb{Z}$ actions and was stated in full generality in OW80. For a survey on the topic of orbit-equivalence, see also Ga00. Now in CFW81 it was shown that any amenable equivalence relation is hyperfinite and any hyperfinite relation is generated by the action of a single transformation. This action is orbit equivalent to the standard odometer action, and we can transfer the hyperfinite exhaustion of the odometer to the underlying equivalence relation (using the orbit equivalence). It follows that bounded hyperfinite exhaustions satisfying the growth condition required in Theorem 1.8 always exist.

2) The proof of Theorem 1.8 is inspired by Lindenstrauss' proof of the Shannon-McMillanBreiman theorem for amenable groups. As such, the analogous growth condition for (tempered) Følner sequences appears there as well, cf. Theorem 1.3 in Li01. In the survey paper We03 Weiss gives a deterministic combinatorial proof of the Shannon-McMillan-Breiman theorem based 
on previous joint work of Ornstein and Weiss. This proof is valid for general tempered Følner sequences even without an additional growth condition and also for non-ergodic actions, see Theorem 6.2 in $\mathrm{We} 03$.

3) The existence of a hyperfinite equivalence relation $\mathcal{R}$ and a cocycle $\alpha$ which satisfy the properties required in Theorem 1.8 for all p.m.p. actions of $\Gamma$ is an interesting question, which at this point has not been fully resolved for all ergodic p.m.p. actions of all countable groups $\Gamma$, as far as we are aware. We shall establish in $\$ 5.3$ below, using an argument developed by A. Kechris for this purpose, that given any countable group, it is possible to find an equivalence relation $\mathcal{R}$ and a cocycle $\alpha: \mathcal{R} \rightarrow \Gamma$, such that the assumption of Theorem 1.8 are satisfied for all strongly mixing actions of $\Gamma$. Furthermore, using a recent result of Vaes and Wahl [VW18, we shall see that for an extensive class of group it is possible to find for any p.m.p. measure-preserving action $(X, \mu)$ of $\Gamma$, a suitable equivalence relation $\mathcal{R}^{X}$ over a product space $(X \times Y, \mu \times \nu)$, and a class injective cocycle $\alpha: \mathcal{R}^{X} \rightarrow \Gamma$ satisfying all the assumptions of Theorem 1.8. However, in this construction, $\mathcal{R}^{X}$ is not derived as an extended relation coming from an equivalence relation over $(Y, \nu)$.

Theorem 1.8 implies the corresponding Shannon-McMillan theorem, asserting convergence of the information functions in $L^{1}$. This result was originally proved by Danilenko and Park in DP02.

Corollary 1.9. DP02, Prop. 1.2] Convergence in Theorem 1.8 holds for $\nu$-almost every $y \in Y$ in the $L^{1}(X, \lambda)$-norm, and also in the $L^{1}(X \times Y, \lambda \times \nu)$-norm.

We do not know whether one can also expect convergence in $L^{1}(Y, \nu)$ for $\lambda$-almost every $x \in X$.

Acknowledgements. The authors would like to thank Lewis Bowen, Brandon Seward and Benjamin Weiss for several enlightening and useful conversations and for their comments on a preliminary version of the present manuscript. The authors are indebted to Alexander Kechris for several insightful observations regarding measurable equivalence relations, and for his permission to include these observations and their proofs in the present paper.

\section{Amenable equivalence Relations}

In this section, we discuss measured Borel equivalence relations which are amenable in the sense of Connes, Feldman and Weiss CFW81. This condition was shown in that paper to be equivalent to hyperfiniteness.

2.1. Measurable equivalence relations. Consider a Borel measurable equivalence relation $\mathcal{R}$ defined over a standard Borel probability space $(Y, \mathcal{B}(Y), \nu)$, namely $\mathcal{R}$ is a Borel measurable subset of $Y \times Y$ with the properties

- $(y, y) \in \mathcal{R}$ for all $y \in Y$,

- if $(y, z) \in \mathcal{R}$, then $(z, y) \in \mathcal{R}$ for all $y, z \in Y$,

- if $(y, z) \in \mathcal{R}$ and $(z, w) \in \mathcal{R}$, then $(y, w) \in \mathcal{R}$ for all $y, z, w \in Y$.

Two points $y$ and $z$ are called $\mathcal{R}$-equivalent points if $(y, z) \in \mathcal{R}$, and the equivalence class is denoted $\mathcal{R}(y)=[y]=[y]_{\mathcal{R}}$. Each $y \in Y$ determines a left and a right fiber in $\mathcal{R}$, given by $\mathcal{R}^{y}=\{(y, z) ; z \mathcal{R} y\} \subset \mathcal{R}$ and $\mathcal{R}_{y}=\{(z, y) ; z \mathcal{R} y\} \subset \mathcal{R}$. We will always assume that for almost every $y$, the fiber $\mathcal{R}^{y}$ (and hence also $\mathcal{R}_{y}$ ) is countable. $c^{y}$ will denote the counting measure on $\mathcal{R}^{y}$, and $c_{y}$ the counting measure on $\mathcal{R}_{y}$. Integrating the counting measures over $Y$, we obtain two $\sigma$ finite (but in general not finite) measures on $\mathcal{R}$, namely $\tilde{\nu}_{l}=\int_{Y} c^{y} d \nu(y)$ and $\tilde{\nu}_{r}=\int_{Y} c_{y} d \nu(y)$. The measure $\nu$ on $Y$ is called $\mathcal{R}$-non-singular if these two measures are equivalent. Note that $\left(\mathcal{R}, \tilde{\nu}_{l}\right)$ is a standard Borel space, and $\pi_{l}: \mathcal{R} \rightarrow Y$ given by $\pi_{l}(y, z)=y$ is a measurable factor map, and similarly for $\tilde{\nu}_{r}$ and $\pi_{r}$. Note that under the coordinate projection $\pi_{l}: \mathcal{R} \rightarrow Y$, the integral above expresses the measure disintegration of $\tilde{\nu}_{l}$ with respect to $\nu$. If $\tilde{\nu}_{l}=\tilde{\nu}_{r}$ then we denote it by $\tilde{\nu}$, and then $\tilde{\nu}$ as well as $\nu$ are called $\mathcal{R}$-invariant, and $\mathcal{R}$ is called a probability-measure-preserving (p.m.p.) equivalence relation. This is the only case we will consider below.

An inner automorphism of the relation $\mathcal{R}$ is a measurable mapping $\phi: Y \rightarrow Y$ which is almost surely bijective with measurable inverse and with its graph $\operatorname{gr}(\phi)$ being contained in $\mathcal{R}$. The 
collection of all inner automorphisms gives rise to a group $\operatorname{Aut}(\mathcal{R})=[\mathcal{R}]$, called the full group of $\mathcal{R}$. A countable subset $\Phi_{0} \in \operatorname{Aut}(\mathcal{R})$ is said to be generating (for $\mathcal{R}$ ) if for $\tilde{\nu}$-almost all $(y, z) \in \mathcal{R}$ there is some $\phi \in \Phi_{0}$ such that $z=\phi(y)$. $\Phi_{0}$ of course generates a countable subgroup of $\operatorname{Aut}(\mathcal{R})$, denoted $\Phi$.

The space Fin $(Y)$ of finite subsets of a Borel space $Y$ is a Borel space in a natural way, using the obvious Borel structure on $\bigcup_{n \in \mathbb{N}} Y^{n} / \operatorname{Sym}(n)$. Measurable mappings of the form $\mathcal{F}: Y \rightarrow \operatorname{Fin}(Y)$ satisfying that for almost every $y$, the set $\mathcal{F}(y)$ consists of finitely many points equivalent to $y$, are called subset functions of $\mathcal{R}$. The possibility that $\mathcal{F}(y)=\phi$ is the empty set is allowed. We consider two subset functions $\mathcal{T}, \mathcal{S}$ to be equal if the set $\{y \mid \mathcal{S}(y) \neq \mathcal{T}(y)\}$ has zero measure. We write $\mathcal{S} \subset \mathcal{T}$ if $\mathcal{S}(y) \subset \mathcal{T}(y)$ for $\nu$-almost every $y$. Subset functions can be composed with each other, inverted, and subtracted from each other. We refer to BN13a and BN13b] for a full discussion, and recall here the following definitions.

$$
\begin{aligned}
\mathcal{S} \circ \mathcal{T}(y) & :=\bigcup_{z \in \mathcal{T}(y)} \mathcal{S}(z), \\
\mathcal{T}^{-1}(y) & :=\{z \in[y] \mid y \in \mathcal{T}(z)\}, \\
(\mathcal{S} \backslash \mathcal{T})(y) & :=\mathcal{S}(y) \backslash \mathcal{T}(y) .
\end{aligned}
$$

A finite non-empty set $D \subset \operatorname{Aut}(\mathcal{R})$ gives rise to the subset function $\mathcal{D}(y)=\{\phi(y) ; \phi \in D\}$. Given a subset function $\mathcal{T}$ and a finite set $D \subset \operatorname{Aut}(\mathcal{R}), \mathcal{D} \circ \mathcal{T}$ is defined as above, and is given by

$$
\mathcal{D} \circ \mathcal{T}(y):=\bigcup_{\phi \in D} \phi(\mathcal{T}(y)) \text {. }
$$

We will also use the notation $D \circ \mathcal{T}$ for this expression. A subset $\mathcal{K} \subseteq \mathcal{R}$ is said to be bounded if

$$
\|\mathcal{K}\|:=\operatorname{ess-sup}_{y} \max \left\{\left|\mathcal{K}_{y}\right|,\left|\mathcal{K}^{y}\right|\right\}<\infty,
$$

where $\mathcal{K}_{y}:=\{z \in[y] \mid(z, y) \in \mathcal{K}\}$ and $\mathcal{K}^{y}:=\{z \in[y] \mid(y, z) \in \mathcal{K}\}$. Analogously, we say that a subset function $\mathcal{T}$ is bounded, if $\|\mathcal{T}\|:=\operatorname{ess}_{-\sup _{y}} \max \left\{|\mathcal{T}(y)|,\left|\mathcal{T}^{-1}(y)\right|\right\}$ is finite.

2.2. Hyperfiniteness and amenability. The relation $\mathcal{R}$ is called hyperfinite if there exists a sequence $\left(\mathcal{R}_{n}\right)$ consisting of subrelations $\mathcal{R}_{n} \subset \mathcal{R}$, where each $\mathcal{R}_{n}$ has finite classes, satisfying

$$
\mathcal{R}_{n} \subseteq \mathcal{R}_{n+1} \quad, \quad \mathcal{R}=\bigcup_{n=1}^{\infty} \mathcal{R}_{n}
$$

We refer to such a sequence as a hyperfinite exhaustion of $\mathcal{R}$. Note that each $\mathcal{R}_{n}$ is a subset function as defined above. If each $\mathcal{R}_{n}$ is a bounded subset function we will call the hyperfinite exhaustion a bounded hyperfinite exhaustion. Recall that it was proved by Connes, Feldman and Weiss [CFW81, Thm. 10] that $\mathcal{R}$ being amenable is equivalent to $\mathcal{R}$ being hyperfinite.

Let us note that hyperfinite exhaustions are asymptotically invariant under inner automorphisms of finite rank, in the following sense (see also [BN13a, BN13b).

Proposition 2.1. If $\left(\mathcal{R}_{n}\right)$ is a bounded hyperfinite exhaustion of the relation $\mathcal{R}$, then there exists an increasing sequence of finite subgroups $\Phi_{n} \subseteq \operatorname{Aut}(\mathcal{R}), n \geq 1$, such that $\Phi:=\bigcup_{n>1} \Phi_{n}$ is generating for $\mathcal{R}$ and for all $n \in \mathbb{N}$ and $\phi \in \Phi_{n}$, the graph $\operatorname{gr}(\phi)$ is contained in $\mathcal{R}_{n}$.

In particular, for every $\phi \in \Phi$, there is an $n_{0} \in \mathbb{N}$ such that $\mathcal{R}_{n}(y) \triangle \phi\left(\mathcal{R}_{n}(y)\right)=\emptyset$ for $\nu$-almost every $y \in Y$ and every $n \geq n_{0}$.

Proof. Let $\mathcal{T}$ be any equivalence subrelation of $\mathcal{R}$ with finite classes of bounded size. We can divide $Y$ to finitely many measurable $\mathcal{T}$-invariant sets where the size of $\mathcal{T}(y)$ is fixed, and we can restrict $\mathcal{T}$ to one of them. Without loss of generality we can thus assume that $\mathcal{T}$ has classes of fixed size $N$ in $Y$. As is well known (see e.g. [FM77, §4], or [CFW81, Lem. 3b]) the factor space $Y / \mathcal{T}$ consisting of equivalence classes of $\mathcal{T}$ is a standard Borel space and admits measurable sections $J_{1}, \ldots, J_{N}: Y / \mathcal{T} \rightarrow Y$ such that $\left\{J_{i}(\mathcal{T}(y)) ; 1 \leq i \leq N\right\}=[y]_{\mathcal{T}}=\mathcal{T}(y)$ for almost every $y$. Define the cyclic permutation $\sigma_{\mathcal{T}(y)}$ given by $J_{1}(\mathcal{T}(y)) \mapsto J_{2}(\mathcal{T}(y)) \mapsto \cdots J_{N}(\mathcal{T}(y)) \mapsto J_{1}(\mathcal{T}(y))$ in each class, whose cycle length is $N$. Denote by $\phi_{\mathcal{T}}$ the map on $Y$ which coincides with $\sigma_{\mathcal{T}(y)}$ on each class $\mathcal{T}(y)$, and denote the cyclic group generated by $\phi_{\mathcal{T}}$ by $\Phi_{\mathcal{T}}$. Then $\phi_{\mathcal{T}}$ is measurable and 
constitutes an inner automorphism of $\mathcal{R}$ which leaves invariant almost every class of the relation $\mathcal{T}$, and the group $\Phi_{\mathcal{T}}$ generates the relation $\mathcal{T}$.

Applying this procedure to each of the finite bounded relations $\mathcal{R}_{n}$ and taking the union of the corresponding groups, the stated result follows.

\section{Pointwise COVERing Lemmas}

We now establish pointwise decomposition results for subset functions which will provide the central tool for proving the Shannon-McMillan-Breiman theorem in the next section. Our main lemma is motivated by the techniques developed by Lindenstrauss for the proof of the corresponding covering lemma for tempered Følner sequences in amenable groups (see [Li01, Lem. 2.4]). However, working with hyperfinite exhaustions, we are able to avoid two material technicalities:

- we do not need to use an auxiliary random parameter in order to be able to choose the desired coverings with high probability;

- we are able to produce strictly disjoint coverings, and the discussion of $\delta$-disjointness (see Li01, Lem. 2.6, 2.7]) becomes unnecessary.

Instead, we exploit the disjointness properties inherent in a sequence of nested equivalence relations, but nevertheless, the proof of pointwise almost sure convergence is still quite difficult and technical.

As usual, $\mathcal{R}$ will denote a p.m.p amenable equivalence relation over $(Y, \nu)$ with $\tilde{\nu}$ denoting the invariant measure on $\mathcal{R}$, and $\left(\mathcal{R}_{n}\right)$ will denote a bounded hyperfinite exhaustion for $\mathcal{R}$.

We start with the following elementary covering (and disjointification) lemma.

Proposition 3.1. Let $N, L \in \mathbb{N}_{\geq 1}$ with $N<L$ and consider an arbitrary finite sequence of subset functions $\mathcal{B}_{j} \subseteq \mathcal{R}_{L}, 1 \leq j \leq \bar{N}$. Further, for $y \in Y$, consider a collection of classes (of the relations $\mathcal{R}_{n}$ where $\left.1 \leq n \leq N\right)$ given by

$$
\mathfrak{F}(y):=\left\{\mathcal{R}_{n(j)}(w) \mid w \in \mathcal{B}_{j}(y), 1 \leq j \leq N\right\} .
$$
that

Then, for a.e. $y \in Y$, we can extract from $\mathfrak{F}(y)$ a disjoint subcollection $\mathfrak{S}(y)$ of classes such

$$
\coprod_{C \in \mathfrak{S}(y)} C \supset \bigcup_{j=1}^{N} \mathcal{B}_{j}(y) \text {, and so } \sum_{C \in \mathfrak{S}(y)}|C| \geq\left|\bigcup_{j=1}^{N} \mathcal{B}_{j}(y)\right| \text {. }
$$

Proof. Fix $y \in Y$. We note first that for $z_{1}, z_{2} \in \mathcal{R}_{L}(y)$ and $1 \leq j_{1}, j_{2} \leq N$, there are three possibilities for the inclusion relation between $\mathcal{R}_{j_{1}}\left(z_{1}\right)$ and $\mathcal{R}_{j_{2}}\left(z_{2}\right)$ :

- $\mathcal{R}_{j_{1}}\left(z_{1}\right) \cap \mathcal{R}_{j_{2}}\left(z_{2}\right)=\emptyset$,

- $\mathcal{R}_{j_{1}}\left(z_{1}\right) \subseteq \mathcal{R}_{j_{2}}\left(z_{2}\right)$, or

- $\mathcal{R}_{j_{1}}\left(z_{1}\right) \supseteq \mathcal{R}_{j_{2}}\left(z_{2}\right)$.

Any collection $\mathfrak{F}(y)$ of sets with the latter property has the property that the union of its constituents has a unique representation as a disjoint union of some of the constituents. To find this representation explicitly, namely to choose the subcollection $\mathfrak{S}(y)$, enumerate all the elements in $\mathfrak{F}(y)$ and give them distinct labels collected in an index set $\mathcal{I}$. Then run the following checking algorithm.

(1) $\operatorname{Set}^{*}(y)=\emptyset, \mathcal{I}^{*}=\mathcal{I}$.

(2) Check an arbitrary class $C \in \mathfrak{F}(y)$ with its corresponding label being contained in $\mathcal{I}^{*}$.

(3) Given $C$, there are two possibilities:

(A) Either for all $1 \leq j \leq N$ and $z \in \mathcal{B}_{j}(y)$ such that $\mathcal{R}_{n(j)}(z) \cap C \neq \emptyset$, we have $C \supseteq \mathcal{R}_{n(j)}(z)$, (B) or there is some $1 \leq j_{1} \leq N, z_{1} \in \mathcal{B}_{j_{1}}(y)$ such that $C \subseteq \mathcal{R}_{n\left(j_{1}\right)}\left(z_{1}\right)$ and $C \neq \mathcal{R}_{n\left(j_{1}\right)}\left(z_{1}\right)$.

(4) Only in case of (A), add $C$ to the subcollection $\mathfrak{S}^{*}(y)$. Then remove from $\mathcal{I}^{*}$ all labels corresponding to classes $\mathcal{R}_{n(j)}(z) \in \mathfrak{F}(y)$ being contained in $C$. If the new set $\mathcal{I}^{*}=\emptyset$, jump to step (5), otherwise return to step (2).

(5) We have $\mathcal{I}^{*}=\emptyset$ (meaning all classes have been checked) and we set $\mathfrak{S}(y)=\mathfrak{S}^{*}(y)$. This is the collection we aim for. 
By construction, for a.e. $Y$, the elements $C \in \mathfrak{S}(y)$ are pairwise disjoint. Also, we made sure that for every $1 \leq j \leq N$, every single $b \in \mathcal{B}_{j}(y)$ is contained in some class $C$ taken into $\mathfrak{S}(y)$. This proves the above inequality.

We now prove the main covering lemma, motivated by [Li01, Lemma 2.1].

Lemma 3.2. Fix $0<\delta<1$ and fix an (arbitrary) finite non-empty set $D \subset \Phi$. Then, for sufficiently large $M \in \mathbb{N}$, depending only on $D$ and $\delta$, the following property holds.

Let $\mathcal{T}_{i, j} \subseteq \mathcal{R}_{L}\left(1 \leq i \leq M, 1 \leq j \leq N_{i}\right)$ be an array of subset functions, such that for a.e. $y$, $\mathcal{T}_{i, j}(y)=\mathcal{R}_{n(i, j)}(y)$, where $n(i, j) \leq L$. Assume that for $2 \leq i \leq M$ and every $1 \leq j \leq N_{i}$

$$
\left|\bigcup_{k<i} D \circ\left(\mathcal{T}_{k, *}^{-1} \mathcal{T}_{i, j}\right)\right| \leq(1+\delta)\left|\mathcal{T}_{i, j}\right|
$$

almost surely, where $\mathcal{T}_{k, *}:=\bigcup_{j=1}^{N_{k}} \mathcal{T}_{k, j}$ for $1 \leq k \leq M$.

Then, given another array $\mathcal{B}_{i, j} \subseteq \mathcal{R}_{L}$ for $1 \leq i \leq M$ and $1 \leq j \leq N_{i}$, for $\nu$-almost every $y \in Y$ there are disjoint subcollections

$$
\mathfrak{S}(y) \subseteq\left\{\mathcal{T}_{i, j}(w) \mid w \in \mathcal{B}_{i, j}(y), 1 \leq i \leq M, 1 \leq j \leq N_{i}\right\}
$$

such that

$$
\sum_{C \in \mathfrak{S}(y)}|C| \geq(1-\delta) \min _{1 \leq i \leq M}\left|D \circ \bigcup_{j=1}^{N_{i}} \mathcal{B}_{i, j}(y)\right| .
$$

Proof. Fix a conull set $Y_{0} \subseteq Y$ such that for all $y \in Y_{0}$ the inequality (3.1) is fulfilled. For $i=M$, apply Proposition 3.1 to the subset functions defined as $\mathcal{B}_{j}:=\mathcal{B}_{M, j}$, where $1 \leq j \leq N_{M}$. This way, by passing to another conull subset of $Y_{0}$, for $\nu$-a.e. $y \in Y_{0}$, we obtain a disjoint subcollection

$$
\mathfrak{S}_{M}(y) \subseteq\left\{\mathcal{T}_{M, j}(w) \mid 1 \leq j \leq N_{M}, w \in \mathcal{B}_{M, j}(y)\right\} .
$$

with

$$
\sum_{C \in \mathfrak{S}_{M}(y)}|C| \geq\left|\bigcup_{j=1}^{N_{M}} \mathcal{B}_{M, j}(y)\right|
$$

Proceeding iteratively, for $i<M$, we set

$$
\tilde{\mathcal{B}}_{i, j}(y):=\mathcal{B}_{i, j}(y) \backslash \bigcup_{l>i} \mathcal{T}_{i, j}^{-1} \circ\left(\bigcup \mathfrak{S}_{l}\right)(y),
$$

where $1 \leq j \leq N_{i}$ and $\bigcup \mathfrak{S}_{l}$ denotes the union over all sets in $\mathfrak{S}_{l}$. Applying Proposition 3.1 again, this time to the subset functions defined as $\mathcal{B}_{j}:=\tilde{\mathcal{B}}_{i, j}, 1 \leq j \leq N_{i}$ gives a subcollection

$$
\mathfrak{S}_{i}(y) \subseteq\left\{\mathcal{T}_{i, j}(w), 1 \leq j \leq N_{i}, w \in \tilde{\mathcal{B}}_{i, j}(y)\right\}
$$

with the corresponding covering property, namely

$$
\sum_{C \in \mathfrak{S}_{i}(y)}|C| \geq\left|\bigcup_{j=1}^{N_{i}} \tilde{\mathcal{B}}_{i, j}(y)\right| .
$$

Having constructed $\mathfrak{S}_{i}$ for all $1 \leq i \leq M$, we finally set $\mathfrak{S}:=\bigcup_{i=1}^{M} \mathfrak{S}_{i}$. By construction, $\mathfrak{S}$ is a disjoint collection of sets. We are going to show

$$
\sum_{i=m}^{M} \sum_{C \in \mathfrak{S}_{i}(y)}|C| \geq \min \left\{1-\delta,(M-m+1) \frac{\delta^{2}}{|D|}\right\} \cdot \min _{m \leq i \leq M}\left|D \circ \bigcup_{j=1}^{N_{i}} \mathcal{B}_{i, j}(y)\right|
$$

for all $1 \leq m \leq M$ and $\nu$-almost every $y \in Y$. The proof is by induction on $m=M, \ldots, 1$, starting with $M$. By the first case in our argument above,

$$
\sum_{C \in \mathfrak{S}_{M}(y)}|C| \geq\left|\bigcup_{j=1}^{N_{M}} \mathcal{B}_{M, j}(y)\right| \geq \frac{1}{|D|}\left|D \circ \bigcup_{j=1}^{N_{M}} \mathcal{B}_{M, j}(y)\right|
$$


which shows the validity of an inequality which is in fact stronger than 3.2 In order to show the claim for $m<M$, we assume that for the element $y \in Y$ under consideration,

$$
\sum_{l>m} \sum_{C \in \mathfrak{S}_{l}(y)}|C|<(1-\delta) \min _{m \leq i \leq M}\left|D \circ \bigcup_{j=1}^{N_{i}} \mathcal{B}_{i, j}(y)\right|
$$

since, otherwise, there is nothing to prove. By construction of the collection $\mathfrak{S}_{m}(y)$, we have

$$
\sum_{C \in \mathfrak{S}_{m}(y)}|C| \geq\left|\bigcup_{j=1}^{N_{m}} \tilde{\mathcal{B}}_{m, j}(y)\right|
$$

It follows from

$$
\tilde{\mathcal{B}}_{m, j}(y)=\mathcal{B}_{m, j}(y) \backslash \bigcup_{l>m} \bigcup_{C \in \mathfrak{S}_{l}(y)} \mathcal{T}_{m, j}^{-1} C
$$

that

$$
\left(D \circ \bigcup_{j=1}^{N_{m}} \tilde{\mathcal{B}}_{m, j}\right)(y) \supseteq\left(D \circ \bigcup_{j=1}^{N_{m}} \mathcal{B}_{m, j}\right)(y) \backslash \bigcup_{l>m} \bigcup_{C \in \mathfrak{S}_{l}(y)} D \circ\left(\bigcup_{j=1}^{N_{m}} \mathcal{T}_{m, j}^{-1} C\right) .
$$

Recall that by assumption (3.1), we have

$$
\left|D \circ\left(\bigcup_{j=1}^{N_{m}} \mathcal{T}_{m, j}^{-1} C\right)\right| \leq(1+\delta)|C|
$$

for a.e. $y \in Y_{0}$, where $C \in \bigcup_{l>m} \mathfrak{S}_{l}(y)$. Hence, by (3.5),

$$
\begin{aligned}
\left|\bigcup_{j=1}^{N_{m}} \tilde{\mathcal{B}}_{m, j}(y)\right| & \geq \frac{1}{|D|}\left|\left(D \circ \bigcup_{j=1}^{N_{m}} \tilde{\mathcal{B}}_{m, j}\right)(y)\right| \\
& \geq \frac{1}{|D|}\left(\left|D \circ \bigcup_{j=1}^{N_{m}} \mathcal{B}_{m, j}(y)\right|-(1+\delta) \sum_{l>m} \sum_{C \in \mathfrak{S}_{l}}|C|\right) .
\end{aligned}
$$

With (3.4) we obtain by taking into account (3.3)

$$
\begin{aligned}
\sum_{C \in \mathfrak{S}_{m}}|C| & \geq \frac{1}{|D|}\left|D \circ \bigcup_{j=1}^{N_{m}} \mathcal{B}_{m, j}(y)\right|-\frac{1+\delta}{|D|} \sum_{l>m} \sum_{C \in \mathfrak{S}_{l}}|C| \\
& \geq(1-(1+\delta)(1-\delta)) \frac{1}{|D|} \min _{m \leq i \leq M}\left|D \circ \bigcup_{j=1}^{N_{i}} \mathcal{B}_{i, j}(y)\right| \\
& =\frac{\delta^{2}}{|D|} \min _{m \leq i \leq M}\left|D \circ \bigcup_{j=1}^{N_{i}} \mathcal{B}_{i, j}(y)\right| .
\end{aligned}
$$

Consequently,

$$
\begin{aligned}
\sum_{l \geq m} \sum_{C \in \mathfrak{S}_{l}}|C| & \geq \sum_{l>m} \sum_{C \in \mathfrak{S}_{l}}|C|+\frac{\delta^{2}}{|D|} \min _{m \leq i \leq M}\left|D \circ \bigcup_{j=1}^{N_{i}} \mathcal{B}_{i, j}(y)\right| \\
& \geq\left((M-(m+1)+1) \frac{\delta^{2}}{|D|}+\frac{\delta^{2}}{|D|}\right) \min _{m \leq i \leq M}\left|D \circ \bigcup_{j=1}^{N_{i}} \mathcal{B}_{i, j}(y)\right| .
\end{aligned}
$$

Hence, the proof of the inequality (3.2) is complete. Finally, choosing $M$ large enough such that

$$
\frac{(M-1) \delta^{2}}{|D|} \geq(1-\delta)
$$

concludes the proof of the lemma. 


\section{Proof of the Shannon-McMillan-Breiman theorem}

The goal of this section is to prove Theorem 1.8, as well as Corollary 1.9, To this end, we adapt the overall strategy given in Li01, Section 4] to the situation of amenable equivalence relations. As usual, $\mathcal{R}$ is p.m.p. and hyperfinite, $\Gamma$ is countable and $\alpha: \mathcal{R} \rightarrow \Gamma$ a class injective measurable cocycle. Recall that for a p.m.p. group action $\Gamma \curvearrowright(X, \lambda)$, the extended equivalence relation $\mathcal{R}^{X}$ over $(X \times Y, \lambda \times \nu)$ is defined by the condition

$$
\left((x, y),\left(x^{\prime}, y^{\prime}\right)\right) \in \mathcal{R}^{X} \Longleftrightarrow y \mathcal{R} y^{\prime} \text { and } x=\alpha\left(y, y^{\prime}\right) x^{\prime},
$$

When the measure $\nu$ is $\mathcal{R}$-invariant, it follows that $\lambda \times \nu$ is $\mathcal{R}^{X}$-invariant, since the $\Gamma$-action on $X$ preserves $\lambda$. The projection map $\pi: \mathcal{R}^{X} \rightarrow \mathcal{R}$ given by $(x, y) \rightarrow y$ is injective when restricted to the $\mathcal{R}^{X}$-equivalence class of $(x, y)$, for almost all $(x, y) \in \mathcal{R}^{X}$. Further, it is well known that an extension of an amenable action is amenable, and thus in particular if $\mathcal{R}$ is amenable, so is $\mathcal{R}^{X}$. But since the extension is class-injective, in fact every hyperfinite exhaustion $\left(\mathcal{R}_{n}\right)$ of $\mathcal{R}$ can be canonically lifted to a hyperfinite exhaustion $\left(\mathcal{R}_{n}^{X}\right)$ of $\mathcal{R}^{X}$, via $\mathcal{R}_{n}^{X}((x, y))=\left\{(\alpha(z, y) x, z) ; z \in \mathcal{R}_{n}(y)\right\}$. Note that if $\left(\mathcal{R}_{n}\right)$ is a bounded hyperfinite exhuastion, then so is $\left(\mathcal{R}_{n}^{X}\right)$, with the same bounds on the equivalence classes.

For a given hyperfinite exhaustion $\left(\mathcal{R}_{n}\right)$ for $\mathcal{R}$, we define a set $\Phi \subseteq \operatorname{Aut}(\mathcal{R})$ satisfying the conclusions of Proposition 2.1. Then, every $\phi \in \Phi$ can be extended naturally to an inner automorphism $\phi^{X} \in \operatorname{Aut}\left(\mathcal{R}^{X}\right)$ by setting

$$
\phi^{X}((x, y)):=(\alpha(\phi(y), y) x, \phi(y)) .
$$

For a subset $D \subseteq \Phi$, we write $D^{X}$ for the set $\left\{\phi^{X} \mid \phi \in D\right\}$. Note that by definition, the set $\Phi^{X}:=\left\{\phi^{X} \mid \phi \in \Phi\right\}$ is generating for the relation $\mathcal{R}^{X}$. Clearly, if $\phi$ preserves the classes of $\mathcal{R}_{n}$ almost surely, then $\phi^{X}$ preserves the classes of $\mathcal{R}_{n}^{X}$ almost surely.

Let us now recall the concept of ergodicity for a measure preserving equivalence relation. Let $\mathcal{Z}$ be such a relation over a probability space $(Z, \eta)$. A subset $A \subseteq Z$ will be called $\mathcal{Z}$-invariant if

$$
(A \times Z) \cap \mathcal{Z}=(A \times A) \cap \mathcal{Z} .
$$

The relation $\mathcal{Z}$ is ergodic if every $\mathcal{Z}$-invariant set $A \subseteq Z$ satisfies $\eta(A) \in\{0,1\}$. From now on, we will always assume that the relation $\mathcal{R}^{X}$ is ergodic. For sufficient conditions guaranteeing this property, we refer to the discussion in $\$ 5$ below.

One crucial ingredient for the proofs of Theorem 1.8 and Corollary 1.9 is the pointwise ergodic theorem. A general form of it being valid for suitable asymptotically invariant sequences of subset functions in an amenable equivalence relation was established in [BN13b, Thm. 2.1]. Since we restrict our discussion here to hyperfinite exhaustions, we will state a less general but easily accessible special case which is sufficient for our purposes. Indeed, the following fact is an immediate consequence of the martingale convergence theorem.

Theorem 4.1. Let $\mathcal{Z}$ be an ergodic, p.m.p. equivalence relation over a probability space $(Z, \eta)$. Let $\left(\mathcal{Z}_{n}\right)$ be a bounded hyperfinite exhaustion for $\mathcal{Z}$.

Then for all $f \in L^{1}(Z, \eta)$, we have

$$
\lim _{n \rightarrow \infty}\left|\mathcal{Z}_{n}(z)\right|^{-1} \sum_{w \in \mathcal{Z}_{n}(z)} f(w)=\int_{Z} f(w) d \eta(w)
$$

for $\eta$-almost every $z \in Z$.

We now turn to establish some preliminary lemmas that will be used below. The first step is a straightforward consequence of the ergodicity of the relation.

Lemma 4.2. Let $\mathcal{Z}$ be an ergodic p.m.p. equivalence relation over a probability $(Z, \eta)$ along with a countable set $\Phi$ of inner automorphisms generating $\mathcal{Z}$. Then, for every $\delta>0$, and every set $A \subseteq Z$ with $\eta(A)>0$, there is a finite set $D \subset \Phi$ such that

$$
\eta(D \circ A) \geq 1-\delta \text {. }
$$


Proof. Let $A \subseteq Z$ be measurable with $\eta(A)>0$. Assume that there is some $\delta_{0}>0$ such that for all finite collections $D \subset \Phi$, we have $\eta(D \circ A)<1-\delta_{0}$. We define $\bar{A}:=\Phi \circ A=\bigcup_{\phi \in \Phi} \phi(A)$. This set is invariant under the relation $\mathcal{Z}$. To see this, consider $z \in Z, \phi \in \Phi$ and $a \in A$ such that $(\phi(a), z) \in \mathcal{Z}$. Since $\Phi$ is generating, there is a further inner automorphism $\phi^{\prime} \in \Phi$ such that $z=\phi^{\prime} \phi(a)$. We conclude that $z \in \Phi \circ A$ and thus,

$$
(\bar{A} \times Z) \cap \mathcal{Z}=(\bar{A} \times \bar{A}) \cap \mathcal{Z} .
$$

Since $\Phi$ is countable, it follows $\eta(\bar{A}) \leq 1-\delta_{0}$ by our assumption. Now $\bar{A}$ is an invariant set, hence by ergodicity of the relation, we obtain $\eta(\bar{A})=0$. However, since every inner automorphism preserves the measure, we have $\eta(\bar{A}) \geq \eta(A)>0$. This is a contradiction.

We need the following combinatorial covering lemma, which establishes the measured equivalence relation version of a covering lemma originally formulated for Følner sequences, see OW83, Lem. 1] and [Li01, Lem. 4.2].

Lemma 4.3. Let $\mathcal{R}$ be a p.m.p. equivalence relation over $(Y, \nu)$, and let $\left(\mathcal{R}_{n}\right)$ be a bounded hyperfinite exhaustion satisfying $\lim _{n \rightarrow \infty} \operatorname{ess}_{\inf _{y}}\left|\mathcal{R}_{n}(y)\right|=\infty$. Then, for every $\eta>0$, there is some $\ell \in \mathbb{N}$ such that the following holds.

Suppose that to $y \in Y$ there corresponds a finite increasing sequence $\left(k_{i}\right), 1 \leq i \leq r(y)$ of integers (depending on $y$ ) with $\left|\mathcal{R}_{k_{1}}(z)\right| \geq \ell$ for almost every $z \in Y$. Then, there is $n_{0} \in \mathbb{N}$ such that for all $n \geq n_{0}$, the number of possible disjoint subcollections $\mathfrak{S}(y)$ of the form

$$
\mathfrak{S}(y) \subseteq\left\{\mathcal{R}_{k_{i}}(c) \mid c \in \mathcal{R}_{n}(y), 1 \leq i \leq r(y)\right\}
$$

is at most $2^{\eta\left|\mathcal{R}_{n}(y)\right|}$.

Proof. Let $\eta>0$. Consider $\ell \in \mathbb{N}, y \in Y$ and an increasing sequence $\left(k_{i}\right), 1 \leq i \leq r(y)$ of integers (depending on $y$ ) with $\left|\mathcal{R}_{k_{1}}(z)\right| \geq \ell$ for all $1 \leq i \leq r(y)$ and almost every $z \in Y$. Since we assume that $\mathfrak{S}(y)$ is a collection of disjoint $\mathcal{R}_{k_{i}}$-classes contained in $\mathcal{R}_{n}(y)$, there is a center set $\mathcal{C}(y) \subseteq \mathcal{R}_{n}(y)$ such that

$$
\mathfrak{S}(y)=\left\{\mathcal{R}_{k_{t(c)}}(c) \mid c \in \mathcal{C}(y)\right\} \text { with } 1 \leq t(c) \leq r(y)
$$

Given $\mathfrak{S}(y)$ (and hence $\mathcal{C}(y)$ ), we define a set of points $\mathcal{N}(y)$ as follows. For $c \in \mathcal{C}(y)$, define $n(c):=i$ as the maximal $1 \leq i \leq t(c)$ such that $\mathcal{R}_{k_{i}}(c) \backslash \mathcal{R}_{k_{i-1}}(c) \neq \emptyset$, where $\mathcal{R}_{0}=\emptyset$ by convention. Then add to the set $\mathcal{N}(y)$ an arbitrary point $p(c) \in \mathcal{R}_{k_{i}}(c) \backslash \mathcal{R}_{k_{i-1}}(c)$. By processing in that way for all $c \in \mathcal{C}(y)$, we obtain a set $\mathcal{N}(y)$ with cardinality at most $|\mathcal{C}(y)|$. We claim that we can uniquely recover $\mathfrak{S}(y)$ from knowing the elements in both sets $\mathcal{C}(y)$ and $\mathcal{N}(y)$. Indeed, given $\mathcal{C}(y)$ and $\mathcal{N}(y)$, by construction, for every $c \in \mathcal{C}(y)$, there is a minimal (and thus unique) $1 \leq t(c) \leq r(y)$ such that $\mathcal{R}_{k_{t(c)}}(c) \cap \mathcal{N}(y) \neq \emptyset$. Hence, the number of possible disjoint subcollections must be bounded by the number of choices for the two sets $\mathcal{C}(y)$ and $\mathcal{N}(y)$. Since the sizes of all classes involved are uniformly bounded from below by $\ell$, the cardinality of every $\mathcal{C}(y)$ and $\mathcal{N}(y)$ is bounded from above by $\left\lceil\left|\mathcal{R}_{n}(y)\right| / \ell\right\rceil$. In light of that, we need to bound the expression

$$
\left(\left\lceil\left|\mathcal{R}_{n}(y)\right| / \ell\right\rceil+1\right)^{2}\left(\begin{array}{c}
\left|\mathcal{R}_{n}(y)\right| \\
\left\lceil\left|\mathcal{R}_{n}(y)\right| / \ell\right\rceil
\end{array}\right)^{2} .
$$

To do so, we use the entropy formula for the Stirling approximation. For this purpose, we define

$$
E(\ell):=\frac{1}{\ell} \log \ell+\left(1-\frac{1}{\ell}\right) \log \left(1-\frac{1}{\ell}\right)^{-1} .
$$

By Stirling's approximation (see e.g. [FS08], Example VIII.10), for large enough $n \in \mathbb{N}$, we get

$$
\left(\begin{array}{c}
\left|\mathcal{R}_{n}(y)\right| \\
\left\lceil\left|\mathcal{R}_{n}(y)\right| / \ell\right\rceil
\end{array}\right)^{2} \leq\left(\exp \left(E(\ell / 2)\left|\mathcal{R}_{n}(y)\right|\right)\right)^{2} \leq 2^{4 E(\ell / 2)\left|\mathcal{R}_{n}(y)\right|} .
$$

Now increasing $n$ if necessary, we can make sure that

$$
\left(\left\lceil\mathcal{R}_{n}(y) / \ell\right\rceil+1\right)^{2}\left(\begin{array}{c}
\left|\mathcal{R}_{n}(y)\right| \\
\left\lceil\left|\mathcal{R}_{n}(y)\right| / \ell\right\rceil
\end{array}\right)^{2} \leq 2^{5 E(\ell / 2)\left|\mathcal{R}_{n}(y)\right|} .
$$


Since $E(\ell) \rightarrow 0$ as $\ell \rightarrow \infty$, we can find some $\ell$ such that $E(\ell / 2)<\eta / 5$, and this completes the proof of the claim.

We are ready to prove the main lemma of this section. It is motivated by Lemma 4.3 in [Li01] and provides an analog of it for hyperfinite exhaustions.

Lemma 4.4. Let $\left(B_{k}\right)$ be a sequence of measurable sets in $X \times Y$ such that

$$
\lambda \times \nu\left(\bigcap_{k=1}^{\infty} \bigcup_{j \geq k} B_{j}\right)>0
$$

Then, for every $\delta>0$ and $\lambda \times \nu$-a.e. $(x, y) \in X \times Y$, there is $n(x, y) \in \mathbb{N}$ for which the following holds true: for each $n \geq n(x, y)$, there is a disjoint collection of subsets of $\mathcal{R}_{n}(y)$

$$
\mathfrak{S}=\left\{\mathcal{R}_{k_{i}}\left(b_{i}\right) \mid 1 \leq i \leq r\right\}
$$

with $b_{i} \in \mathcal{R}_{n}(y)$ and $1 \leq k_{i}<n(x, y)$, such that

(i) $\left(\alpha\left(b_{i}, y\right) x, b_{i}\right) \in B_{k_{i}}$ for all $i$,

(ii) $\sum_{C \in \mathfrak{S}}|C| \geq(1-\delta)\left|\mathcal{R}_{n}(y)\right|$.

Proof. Let $\delta>0$. We set

$$
B^{*}:=\bigcap_{k=1}^{\infty} \bigcup_{j \geq k} B_{j}
$$

By assumption, $(\lambda \times \nu)\left(B^{*}\right)>0$. Given the hyperfinite exhaustion $\left(\mathcal{R}_{n}\right)$, we fix $\Phi^{X} \subseteq \operatorname{Aut}\left(\mathcal{R}^{X}\right)$ satisfying the conclusions of Proposition 2.1. Since the extended relation $\mathcal{R}^{X}$ is ergodic, and since the set $\Phi^{X}$ is generating for $\mathcal{R}^{X}$, we can use Lemma 4.2 in order to find a finite set $D \subset \Phi$ such that the lifted automorphisms $D^{X} \subseteq \Phi^{X}$ satisfy

$$
\lambda \times \nu\left(D^{X} \circ B^{*}\right) \geq 1-\delta / 10 .
$$

Thus clearly $\lambda \times \nu\left(B^{*}\right) \geq(1-\delta / 10) /|D|$. Further, choose $M \in \mathbb{N}$ such that Lemma 3.2 holds true for the fixed parameters $\delta$ and $D$ for the relation $\mathcal{R}$.

The next step is to construct two finite increasing integer sequences $\left(m_{i}\right),\left(N_{i}\right), 1 \leq i \leq M$, according to the following algorithm.

(1) Define $m_{1}:=1$.

(2) If $m_{i}$ has been chosen, then determine $N_{i}$ large enough such that

$$
\lambda \times \nu\left(B^{*} \backslash \bigcup_{j=m_{i}}^{m_{i}+N_{i}} B_{j}\right)<\frac{\delta \cdot \lambda \times \nu\left(B^{*}\right)}{10 M|D|} .
$$

(3) Further, if $N_{i}$ has been chosen, choose $m_{i+1}$ large enough such that for every $l \geq m_{i+1}$, we get

$$
\bigcup_{j<m_{i}+N_{i}} D \circ \mathcal{R}_{j}^{-1} \mathcal{R}_{l} \subseteq \mathcal{R}_{l}
$$

This is possible by Proposition 2.1 and since $D \subset \Phi$. In fact $D \circ \mathcal{R}_{n}=\mathcal{R}_{n}$ for all sufficiently large $n$. So it suffices that $m_{i+1}>m_{i}+N_{i}$ and also that $\mathcal{R}_{m_{2}}$ (and hence each $\mathcal{R}_{m_{i+1}}$ ) is invariant under $D$.

With the sequences $\left(m_{i}\right),\left(N_{i}\right)$ at our disposal, we define

$$
\tilde{B}^{*}:=\bigcap_{i=1}^{M} \bigcup_{j=m_{i}}^{m_{i}+N_{i}} B_{j}
$$

Clearly

and so

$$
\lambda \times \nu\left(B^{*} \backslash \tilde{B}^{*}\right) \leq \sum_{i=1}^{M} \lambda \times \nu\left(B^{*} \backslash \bigcup_{j=m_{i}}^{m_{i}+N_{i}} B_{j}\right)<\frac{\delta \cdot \lambda \times \nu\left(B^{*}\right)}{10|D|}
$$

$$
\lambda \times \nu\left(D^{X} \circ B^{*} \backslash D^{X} \circ \tilde{B}^{*}\right)<\frac{\delta \cdot \lambda \times \nu\left(B^{*}\right)}{10} .
$$


Consequently,

$$
\lambda \times \nu\left(D^{X} \circ \tilde{B}^{*}\right) \geq \nu\left(D^{X} \circ B^{*}\right)-\frac{\delta \cdot \lambda \times \nu\left(B^{*}\right)}{10} \geq 1-\frac{\delta}{10}-\frac{\delta \cdot \lambda \times \nu\left(B^{*}\right)}{10} \geq 1-\frac{\delta}{5} .
$$

Now by the pointwise ergodic theorem (Theorem 4.1), for $\lambda \times \nu$-a.e. $(x, y)$, we can define $n(x, y)$ as the smallest integer value greater than $m_{M}+N_{M}$ such that for all $n \geq n(x, y)$, we have

$$
\mathcal{A}\left(n, \mathbf{1}_{D^{X} \circ \tilde{B}^{*}}\right)(x, y):=\left|\mathcal{R}_{n}(y)\right|^{-1} \sum_{z \in \mathcal{R}_{n}(y)} \mathbf{1}_{D^{X} \circ \tilde{B}^{*}}((\alpha(z, y) x, z))>1-\frac{\delta}{4} .
$$

We fix some pair $(x, y)$ satisfying this condition, as well as $n \geq n(x, y)$. For $1 \leq i \leq M$ and $1 \leq j \leq N_{i}$, set $\mathcal{T}_{i, j}:=\mathcal{R}_{m_{i}+j-1}$ and

$$
A_{i, j}(y):=\left\{b \in \mathcal{R}_{n}(y) \mid(\alpha(b, y) x, b) \in B_{m_{i}+j-1}\right\} .
$$

By step (3) of the algorithm and since $n>m_{M}+N_{M}$, we have $D \circ \mathcal{R}_{n} \subseteq \mathcal{R}_{n}$. In particular, each $\phi \in D$ gives rise to bijections $\phi: \mathcal{R}_{n}(y) \rightarrow \mathcal{R}_{n}(y)$, for a.e. $y \in Y$.

Applying the transformations in the set $D$ to the sets $A_{i, j}(y)$, we then obtain

$$
\begin{aligned}
D \circ \bigcup_{j=1}^{N_{i}} A_{i, j}(y) & =\bigcup_{\phi \in D}\left\{\phi(b) \mid b \in \mathcal{R}_{n}(y),(\alpha(b, y) x, b) \in \bigcup_{j=1}^{N_{i}} B_{m_{i}+j-1}\right\} \\
& =\left\{b \in \mathcal{R}_{n}(y) \mid \exists \phi \in D, b^{\prime} \in \mathcal{R}_{n}(y): b=\phi\left(b^{\prime}\right),\left(\alpha\left(b^{\prime}, y\right) x, b^{\prime}\right) \in \bigcup_{j=1}^{N_{i}} B_{m_{i}+j-1}\right\} .
\end{aligned}
$$

Together with (4.3), this yields for all $1 \leq i \leq M$ :

$$
\begin{aligned}
\left|\bigcup_{j=1}^{N_{i}} D \circ A_{i, j}(y)\right| & =\left|\left\{b \in \mathcal{R}_{n}(y) \mid \exists \phi \in D: b^{\prime}=\phi^{-1}(b),\left(\alpha\left(b^{\prime}, y\right) x, b^{\prime}\right) \in \bigcup_{j=1}^{N_{i}} B_{m_{i}+j-1}\right\}\right| \\
\quad(\operatorname{using}(4.1)) & =\left|\left\{b \in \mathcal{R}_{n}(y) \mid(\alpha(b, y) x, b) \in D^{X} \circ \bigcup_{j=1}^{N_{i}} B_{m_{i}+j-1}\right\}\right|
\end{aligned}
$$

(since $\tilde{B}^{*}$ is an intersection) $\geq\left|\mathcal{R}_{n}(y)\right| \mathcal{A}\left(n, \mathbf{1}_{D^{X} \circ \tilde{B}^{*}}\right)(x, y)$

(by the pointwise ergodic theorem) $\geq\left(1-\frac{\delta}{4}\right)\left|\mathcal{R}_{n}(y)\right|$.

We finally apply Lemma 3.2 with $\delta / 4$ instead of $\delta$ to the arrays $\mathcal{T}_{i, j}$ and $A_{i, j}(y)$, where $1 \leq i \leq M$ and $1 \leq j \leq N_{i}$. The assumption of the Lemma is indeed satisfied, namely $\mathcal{T}_{i, j}=\mathcal{R}_{m_{i}+j-1}$ satisfy (5.1) by the construction of $m_{2}$, which guarantees $D \circ \mathcal{R}_{m}=\mathcal{R}_{m}$ for all $m \geq m_{2}$. Furthermore, we have just shown that

$$
\min _{1 \leq i \leq M}\left|\bigcup_{j=1}^{N_{i}} D \circ A_{i, j}(y)\right| \geq\left(1-\frac{\delta}{4}\right)\left|\mathcal{R}_{n}(y)\right|
$$

and hence Lemma 3.2 implies that there is a disjoint subcollection

$$
\mathfrak{S}(y) \subseteq\left\{\mathcal{T}_{i, j}(b) \mid b \in A_{i, j}(y)\right\}
$$

with

$$
\sum_{C \in \mathfrak{S}(y)}|C| \geq(1-\delta)\left|\mathcal{R}_{n}(y)\right|
$$

as desired.

The following is an immediate consequence from the previous lemma. 
Lemma 4.5. Let $\left(\mathcal{R}_{n}\right)$ be a bounded hyperfinite exhaustion, and keep the assumptions of the previous lemma. Then, for the number

$$
h:=\operatorname{ess}-\inf _{x, y} \liminf _{n \rightarrow \infty} \frac{\mathcal{J}\left(\mathcal{P}^{\mathcal{R}_{n}(y)}(x)\right)}{\left|\mathcal{R}_{n}(y)\right|},
$$

the following holds true. For every $\delta>0$, each $N \in \mathbb{N}$ and $\lambda \times \nu$-a.e. $(x, y)$, there is a number $n(x, y) \in \mathbb{N}$ such that for all $n \geq n(x, y)$, we can find a disjoint collection (depending on both $x$ and $y)$

$$
\mathfrak{S}=\left\{\mathcal{R}_{k_{i}}\left(b_{i}\right) \mid 1 \leq i \leq r\right\}
$$

such that all $b_{i} \in \mathcal{R}_{n}(y)$ and $N \leq k_{i}<n(x, y)$ and further,

(i) for all $i$,

$$
\frac{\mathcal{J}\left(\mathcal{P}^{\mathcal{R}_{k_{i}}\left(b_{i}\right)}\left(\alpha\left(b_{i}, y\right) x\right)\right)}{\left|\mathcal{R}_{k_{i}}\left(b_{i}\right)\right|} \leq h+\delta,
$$

(ii) $\sum_{C \in \mathfrak{S}}|C| \geq(1-\delta)\left|\mathcal{R}_{n}(y)\right|$.

Proof. We apply the previous Lemma 4.4 to the sequence $\left(\mathcal{R}_{n}\right)_{n \geq N}$ and the sets

$$
B_{k}:=\left\{(x, y) \in X \times Y \mid \frac{\mathcal{J}\left(\mathcal{P}^{\mathcal{R}_{N+k}(y)}(x)\right)}{\left|\mathcal{R}_{N+k}(y)\right|} \leq h+\delta\right\} .
$$

We are now in position to prove the Shannon-McMillan-Breiman Theorem. To do so, we combine the previous results of this section, motivated by the proof of Theorem 1.3 in [Li01].

Proof of Theorem 1.8. As before, we set

$$
h:=\operatorname{essinf}_{x, y} \liminf _{n \rightarrow \infty} \frac{\mathcal{J}\left(\mathcal{P}^{\mathcal{R}_{n}(y)}(x)\right)}{\left|\mathcal{R}_{n}(y)\right|} .
$$

If $h=\infty$, then there is nothing left to show. So assume that $h<\infty$. We show that for a.e. $(x, y)$,

$$
\limsup _{n \rightarrow \infty} \frac{\mathcal{J}\left(\mathcal{P}^{\mathcal{R}_{n}(y)}(x)\right)}{\left|\mathcal{R}_{n}(y)\right|} \leq h .
$$

To this end, fix $\delta>0$. We find $N \in \mathbb{N}$ large enough such that Lemma 4.3 holds for $\eta=\delta$ and $\ell=N$. By the growth assumption on the $\left(\mathcal{R}_{n}\right)$, we find $N_{1} \in \mathbb{N}$ such that for a full measure set of $y$ 's, we have $\left|\mathcal{R}_{k}(z)\right| \geq N$ for all $k \geq N_{1}$ and each $z \in[y]$. We have seen in Lemma 4.5 that for $\lambda \times \nu$-almost every $(x, y)$, there is $n(x, y) \in \mathbb{N}, n(x, y)>N_{1}$, such that for $n>n(x, y)$, we obtain a special subcollection $\mathfrak{S}=\mathfrak{S}(x, y)$ of subsets of $\mathcal{R}_{n}(y)$. Namely, this collection

- is disjoint,

- satisfies $\sum_{C \in \mathfrak{S}}|C| \geq(1-\delta)\left|\mathcal{R}_{n}(y)\right|$,

- Each $C \in \mathfrak{S}$ is of the form $\mathcal{R}_{k}(b)$ with $N_{1} \leq k<n(x, y)$ and $b \in \mathcal{R}_{n}(y)$, which by the choice of $N_{1}$ implies that $\left|\mathcal{R}_{k}(b)\right| \geq N$ for all $k \geq N_{1}$,

- writing $C=\mathcal{R}_{k}(b) \in \mathfrak{S}$, the following holds

$$
\frac{\mathcal{J}\left(\mathcal{P}^{\mathcal{R}_{k}(b)}(\alpha(b, y) x)\right)}{\left|\mathcal{R}_{k}(b)\right|} \leq h+\delta
$$

By increasing $n$ if necessary, due to $\left|\mathcal{R}_{n}(y)\right| \rightarrow \infty$, we can assume that

$$
\left|\mathcal{R}_{n}(y)\right| \geq 8 \delta^{-1}(\log n) .
$$

Let us now fix $x, y, n=n(x, y)$ and $\mathfrak{S}(x, y)=\mathfrak{S}$ satisfying all the conditions stated above.

We first note that since $\mathcal{R}_{n}(y)$ is the disjoint union of the sets $C=\mathcal{R}_{k}\left(b_{C}\right) \in \mathfrak{S}$ and $\mathcal{G}_{n}(y):=$ $\mathcal{R}_{n}(y) \backslash \bigcup_{C \in \mathfrak{S}} \mathcal{R}_{k}\left(b_{C}\right)$, it follows that the partition $\mathcal{P}^{\mathcal{R}_{n}(y)}$ is given by $\left(\bigvee_{C \in \mathfrak{S}} \mathcal{P}^{\mathcal{R}_{k}\left(b_{C}\right)}\right) \vee \mathcal{P}^{\mathcal{G}_{n}(y)}$ Therefore for any point $x \in X$, its $\mathcal{P}^{\mathcal{R}_{n}(y)}$-name arises as the intersection of the $\mathcal{P}^{\mathcal{R}_{k}\left(b_{C}\right)}$-name of $\alpha\left(b_{C}, y\right) x$ where $C=\mathcal{R}_{k}\left(b_{C}\right) \in \mathfrak{S}$, and of the $\mathcal{P}$-name of $\alpha(b, y) x$ for every $b \in \mathcal{G}_{n}(y):=$ $\mathcal{R}_{n}(y) \backslash \bigcup_{C \in \mathfrak{S}} \mathcal{R}_{k}\left(b_{C}\right)$. 
Consider the partition $\mathcal{P}^{\mathcal{R}_{n}(y)}$ (which is finer than each partition $\mathcal{P}^{\mathcal{R}_{k}(b)}$ when $b \in \mathcal{R}_{n}(y)$ ) and define a set of atoms in it which we denote by $K_{n}(x, y)$. Namely, for our fixed $x$, we consider the disjoint collection $\mathfrak{S}(x, y)$ of subsets of $\mathcal{R}_{n}(y)$, and we put an atom of $\mathcal{P}^{\mathcal{R}_{n}(y)}$ in $K_{n}(x, y)$ provided that it arises as the intersection of elements of the partitions $\mathcal{P}^{\mathcal{R}_{k}(b)}$ which satisfy inequality (4.5) (where we allow any $\mathcal{R}_{k}(b)=C \in \mathfrak{S}(x, y)$ ), with elements in the partition $\mathcal{P}^{\mathcal{G}_{n}(y)}$.

Now, for every $\mathcal{R}_{k}(b)=C \in \mathfrak{S}(x, y)$, inequality (4.5) gives a lower bound on the measure of some of the atoms in the partition, and hence an upper bound on their number. It follows that for each such $C$, there are at most $2^{(h+\delta)\left|\mathcal{R}_{k}(b)\right|}$ atoms of $\mathcal{P}^{\mathcal{R}_{k}(b)}$ (namely $\mathcal{P}^{\mathcal{R}_{k}(b)}$-names) for which the inequality (4.5) can hold true.

Consequently, the number of atoms of $\mathcal{P}^{\mathcal{R}_{n}(y)}$ which appear as elements in $K_{n}(x, y)$ is bounded by

$$
\prod_{C \in \mathfrak{S}} 2^{(h+\delta)\left|\mathcal{R}_{k}(b)\right|} \cdot|\mathcal{P}|^{\left|\mathcal{G}_{n}(y)\right|}=\prod_{C \in \mathfrak{S}} 2^{(h+\delta)|C|} \cdot|\mathcal{P}|^{\left|\mathcal{R}_{n}(y) \backslash \cup \mathfrak{S}\right|} .
$$

Since $\mathcal{R}_{n}(y)$ is disjointly $(1-\delta)$-covered by the elements in $\mathfrak{S}$, we conclude that for a fixed $x \in X$, there are at most

$$
2^{(h+\delta)\left|\mathcal{R}_{n}(y)\right|} \cdot|\mathcal{P}|^{\delta\left|\mathcal{R}_{n}(y)\right|}
$$

many elements in $K_{n}(x, y)$.

$K_{n}(x, y)$ depends on both $x$ and $y$, having been constructed using the collection $\mathfrak{S}(x, y)$ of subsets on $\mathcal{R}_{n}(y)$. Now, we define another collection of atoms of $\mathcal{P}^{\mathcal{R}_{m}(y)}$, which we denote by $K_{m}(y)$. It consists of all the atoms in the sets $K_{m}(x, y)$ as $x$ varies in $X$, provided that $n(x, y)$ satisfies the conditions stated above and in addition $n(x, y) \leq m$. By Lemma 4.3 (and the choices for $N$ and $N_{1}$ ), for all $m \geq m(y)$ the number of possibilities for $\mathfrak{S}(x, y)$ is bounded by $2^{\delta\left|\mathcal{R}_{m}(y)\right|}$. Hence, we obtain

$$
\left|K_{m}(y)\right| \leq 2^{(h+2 \delta+\delta \log |\mathcal{P}|)\left|\mathcal{R}_{m}(y)\right|}
$$

We now consider the sets

$$
X_{m}(y):=\left\{x \in X\left|\frac{\mathcal{J}\left(\mathcal{P}^{\mathcal{R}_{m}(y)}(x)\right)}{\left|\mathcal{R}_{m}(y)\right|}>h+3 \delta+\delta \log \right| \mathcal{P} \mid\right\} .
$$

Consider $\bigcup K_{m}(y)$, the union of all $\mathcal{P}^{\mathcal{R}_{m}(y)}$-atoms which belong to $K_{m}(y)$. Then,

$$
\lambda\left(X_{m}(y) \cap \bigcup K_{m}(y)\right) \leq\left|K_{m}(y)\right| 2^{-(h+3 \delta+\delta \log |\mathcal{P}|)\left|\mathcal{R}_{m}(y)\right|} \leq 2^{-\delta\left|\mathcal{R}_{m}(y)\right|} .
$$

It follows from the growth condition (4.6) stated above that

$$
2^{-\delta\left|\mathcal{R}_{m}(y)\right|} \leq 2^{\log m^{-8}} \leq \frac{1}{m^{2}}
$$

for large enough $m$. This implies that $\sum_{m=1}^{\infty} 2^{-\delta\left|\mathcal{R}_{m}(y)\right|}<\infty$. Thus, for $\nu$-almost-every $y \in Y$, we can apply the Borel-Cantelli lemma and obtain that for $\lambda \times \nu$-almost every $(x, y), x \notin X_{m}(y) \cap$ $\bigcup K_{m}(y)$ if $m$ is large enough. On the other hand, we deduce from Lemma 4.5 that for large enough $m$ (depending on $x$ and $y$ ), $x \in \bigcup K_{m}(y)$. This implies that for $\lambda \times \nu$-a.e. $(x, y)$, and $m$ large enough, we must have $x \notin X_{m}(y)$, which means by definition of $X_{m}(y)$ that

$$
\limsup _{m \rightarrow \infty} \frac{\mathcal{J}\left(\mathcal{P}^{\mathcal{R}_{m}(y)}(x)\right)}{\left|\mathcal{R}_{m}(y)\right|} \leq h+3 \delta+\delta \log |\mathcal{P}| .
$$

Letting $\delta \rightarrow 0$ yields (4.4). This establishes almost everywhere convergence, as stated. Since the integrals of $\mathcal{J}\left(\mathcal{P}^{\mathcal{R}_{m}(y)}(x)\right) /\left|\mathcal{R}_{m}(y)\right|$ over $X \times Y$ converge to the orbital entropy of $\mathcal{P}$ by Theorem 1.2, we have $h=h_{\mathcal{P}}^{*}(\alpha)$, as claimed.

We conclude this section with the proof of Corollary 1.9. The proof follows along the lines of the $L^{1}$-convergence case proved in Li01, Thm. 4.1]. 
Proof of Corollary 1.9. By Theorem 1.8, the normalized information function converges pointwise almost surely (w.r.t. the measure $\lambda \times \nu$ ) to $h_{\mathcal{P}}^{*}=h_{\mathcal{P}}^{*}(\alpha)$. Fix $\varepsilon>0$, and for $n \in \mathbb{N}$ and fixed $y \in Y$, define

$$
\mathcal{C}_{n}(y):=\left\{x \mid h_{\mathcal{P}}^{*}-\varepsilon \leq \frac{\mathcal{J}\left(\mathcal{P}^{\mathcal{R}_{n}(y)}(x)\right)}{\left|\mathcal{R}_{n}(y)\right|} \leq h_{\mathcal{P}}^{*}+\varepsilon\right\} .
$$

Integration over $X$ gives

$$
\begin{aligned}
\left(h_{\mathcal{P}}^{*}-\varepsilon\right) \lambda\left(\mathcal{C}_{n}(y)\right) & \leq \int_{\mathcal{C}_{n}(y)} \frac{\mathcal{J}\left(\mathcal{P}^{\mathcal{R}_{n}(y)}(x)\right)}{\left|\mathcal{R}_{n}(y)\right|} d \lambda(x) \\
& \leq \int_{X} \frac{\mathcal{J}\left(\mathcal{P}^{\mathcal{R}_{n}(y)}(x)\right)}{\left|\mathcal{R}_{n}(y)\right|} d \lambda(x) \\
& \leq\left(h_{\mathcal{P}}^{*}+\varepsilon\right) \lambda\left(\mathcal{C}_{n}(y)\right)+\int_{X \backslash \mathcal{C}_{n}(y)} \frac{\mathcal{J}\left(\mathcal{P}^{\mathcal{R}_{n}(y)}(x)\right)}{\left|\mathcal{R}_{n}(y)\right|} d \lambda(x)
\end{aligned}
$$

We define a new measure $\lambda^{*}$ on $X \backslash \mathcal{C}_{n}(y)$ by setting

$$
\lambda^{*}(A):=\frac{\lambda(A)}{\lambda\left(X \backslash \mathcal{C}_{n}(y)\right)} .
$$

Then, since $\mathcal{C}_{n}(y)$ is a disjoint union of atoms of the partition $\mathcal{P}^{\mathcal{R}_{n}(y)}$ of $X$,

$$
\begin{aligned}
\int_{X \backslash \mathcal{C}_{n}(y)} \frac{\mathcal{J}\left(\mathcal{P}^{\mathcal{R}_{n}(y)}(x)\right)}{\left|\mathcal{R}_{n}(y)\right|} d \lambda(x)= & \lambda\left(X \backslash \mathcal{C}_{n}(y)\right) \int_{X \backslash \mathcal{C}_{n}(y)} \frac{\mathcal{J}^{*}\left(\mathcal{P}^{\mathcal{R}_{n}(y)}(x)\right)}{\left|\mathcal{R}_{n}(y)\right|} d \lambda^{*}(x) \\
& -\lambda\left(X \backslash \mathcal{C}_{n}(y)\right) \log \left(\lambda\left(X \backslash \mathcal{C}_{n}(y)\right)\right),
\end{aligned}
$$

where $\mathcal{J}^{*}$ denotes the information function with respect to $\lambda^{*}$. Now since the integral on the right hand side in the inequality above is just the Shannon entropy of the partition $\mathcal{P}^{\mathcal{R}_{n}(y)}$ with respect to the measure $\lambda^{*}$, we arrive at

$$
\int_{X \backslash \mathcal{C}_{n}(y)} \frac{\mathcal{J}\left(\mathcal{P}^{\mathcal{R}_{n}(y)}(x)\right)}{\left|\mathcal{R}_{n}(y)\right|} d \lambda(x) \leq \lambda\left(X \backslash \mathcal{C}_{n}(y)\right) \log |\mathcal{P}|-\lambda\left(X \backslash \mathcal{C}_{n}(y)\right) \log \left(\lambda\left(X \backslash \mathcal{C}_{n}(y)\right)\right) .
$$

Clearly, by Theorem 1.8, for $\nu$-almost every $y \in Y$, the latter expression tends to zero as $n \rightarrow \infty$. Now sending $\varepsilon \rightarrow 0$ yields the first assertion of the claimed statement. For the second statement note that due to the dominated convergence theorem (with dominating function $g(y):=2|\mathcal{P}|$ ) we have

$$
\lim _{n \rightarrow \infty} \int_{Y} \int_{X}\left|\frac{\mathcal{J}\left(\mathcal{P}^{\mathcal{R}_{n}(y)}(x)\right)}{\left|\mathcal{R}_{n}(y)\right|}-h_{\mathcal{P}}^{*}\right| d \lambda(x) d \nu(y)=0 .
$$

This concludes the proof of the corollary.

\section{Amenable Relations, injective CocyCles And ERGodic EXtensions}

5.1. Groups admitting injective cocycles. As usual, Let $(Y, \nu)$ be a probability space, and let $\mathcal{R} \subset Y \times Y$ be a p.m.p. Borel equivalence relation with $\mathcal{R}$-invariant probability measure $\tilde{\nu}$, such that $\mathcal{R}=\bigcup_{n \in \mathbb{N}} \mathcal{R}_{n}$ is hyperfinite, or equivalently, $\mathcal{R}$ is amenable in the sense of CFW81. Let $(X, \lambda)$ be a p.m.p. action of a countable group $\Gamma$ and let $\alpha: \mathcal{R} \rightarrow \Gamma$ be a measurable cocycle. Let $\mathcal{R}^{X}$ denote the extended relation on $(X \times Y, \lambda \times \nu)$.

As note in the introduction, by a result of Danilenko [Da01, Cor. 2.7], given the ingredients just listed the following limit exists

$$
h_{\mathcal{P}}^{*}(\alpha):=\lim _{n \rightarrow \infty} \int_{Y} \frac{h^{\mathcal{P}}\left(\mathcal{R}_{n}\right)(y)}{\left|\mathcal{R}_{n}(y)\right|} d \nu(y) .
$$

A meaningful entropy invariant arises when we assume that the hyperfinite exhaustion is by bounded finite relations, and the cocycle is class injective. As we will see shortly, in fact all countable groups admit a class injective cocycle defined on a p.m.p. ergodic hyperfinite relation, but let us first note some familiar explicit examples for this property. 


\section{1) Amenable groups.}

Let $\Gamma$ be amenable, let $(Y, \nu)$ be any p.m.p. action of $\Gamma$, and assume that the action is essentially free. For example, we can take $Y$ to the Bernoulli action of $\Gamma$ on $\{0,1\}^{\Gamma}$. Let $\mathcal{R}=\mathcal{O}_{\Gamma}$ be the orbit relation of $\Gamma$ on $Y$, and then there is a cocycle $\alpha: \mathcal{R} \rightarrow \Gamma$ given by $\alpha(\gamma y, y)=\gamma$. This cocycle is indeed an injective cocycle on a p.m.p. amenable equivalence relation $\mathcal{R}$, by amenability of $\Gamma$ and freeness of the action. Given a p.m.p. action of $\Gamma$ on a space $(X, \lambda)$, clearly $\Gamma$ acts on the product $(X \times Y, \lambda \times \nu)$, and the orbit relation of $\Gamma$ in the product coincides with the extended relation $\mathcal{R}^{X}$ on $X \times Y$ that we have used throughout the paper. Thus $\alpha: \mathcal{R} \rightarrow \Gamma$ is an injective cocycle defined on a p.m.p. amenable relation.

2) The Maharam extension of the Poisson boundary. For every countable infinite group $\Gamma$, and every generating probability measure $\mu$ on $\Gamma$, the Poisson boundary $B=B(\Gamma, \mu)$ is an amenable action of $\Gamma$, in the sense defined by Zimmer Zi78, or equivalently, in the sense of CFW81. Let $\eta$ denote the stationary measure on $B$, and $r_{\eta}(\gamma, b)=\frac{d \eta \circ \gamma}{d \eta}(b)$ the Radon-Nikodym derivative cocycle of $\eta$, so that $r_{\eta}: \Gamma \times B \rightarrow \mathbb{R}_{+}^{*}$. The Maharam extension of $B$ by the cocycle $r_{\eta}$ is the $\Gamma$-action on $B \times \mathbb{R}$ given by $\gamma(b, t)=\left(\gamma b, t-\log r_{\eta}(\gamma, b)\right)$, and this action preserves the measure $\eta \times \theta$, where $d \theta(t)=e^{t} d t$ and $d t$ is Lebesgue measure on $\mathbb{R}$. This action is again an amenable action of $\Gamma$, being an extension of an amenable action. Let us define $Y=B \times(-\infty, 0)$, and let $\nu$ be the restriction of $\eta \times L$ to $Y$, a finite measure which we normalize to be a probability measure. Then $(Y, \nu)$ is a probability space, and we define the relation $\mathcal{R}$ on it to be the restriction of the orbit relation $\mathcal{O}_{\Gamma}$ defined by $\Gamma$ on $B \times \mathbb{R}$ to the subset $Y$. Thus $\mathcal{R}$ is a p.m.p. Borel equivalence relation with countable classes, since $\Gamma$ is countable and preserves the measure $\eta \times L$. The orbit relation $\mathcal{O}_{\Gamma}$ is an amenable relation, hence it is hyperfinite, and as a result so is its restriction $\mathcal{R}$ to the subset $Y$. Finally, if the action of $\Gamma$ on its Poisson boundary $B$ is essentially free, then we can define a cocycle $\alpha: \mathcal{O}_{\Gamma} \rightarrow \Gamma$, by the formula $\alpha(\gamma(b, t),(b, t))=\gamma$. This cocycle is well-defined since the elements in a $\Gamma$-orbit are in bijective correspondence to the elements of $\Gamma$, by essential freeness. Restricting $\alpha$ to $\mathcal{R}$ we obtain a cocycle from an amenable p.m.p. equivalence relation $\mathcal{R}$ to $\Gamma$. Furthermore, this cocycle is injective in this case, since $\gamma(b, t)=\left(\gamma b, t-\log r_{\eta}(\gamma, b)\right)$, so that if $\gamma \neq \gamma^{\prime}$ then $\alpha((b, t), \gamma(b, t)) \neq \alpha\left((b, t), \gamma^{\prime}(b, t)\right)$.

Clearly, the class of groups admitting a random walk such that the action on the associated Poisson boundary is essentially free is extensive indeed. In fact typically many different random walks on a given non-amenable group $\Gamma$ give rise to Poisson boundaries admitting an essentially free action. It is a remarkable feature of the construction of orbital Rokhlin entropy that it gives one and the same value for the entropy of the $\Gamma$-action on $X$, provided only that this action is ergodic and essentially free, and the value is independent of which cocycle $\alpha: \mathcal{R} \rightarrow \Gamma$ as above was chosen to calculate it.

3) We now note the following result, communicated to us by A. Kechris.

Proposition 5.1. Ke17 For any countable infinite group $\Gamma$, there is a class injective cocycle from a hyperfinite p.m.p ergodic equivalence relation $E$ into $\Gamma$.

Proof. Let $E_{0}$ be the equivalence relation on $2^{\mathbb{N}}$ defined by the synchronous tail relation, namely $x E_{0} y \Longleftrightarrow \exists n \forall m>n\left(x_{m}=y_{m}\right)$. This is a hyperfinite relation consisting of an increasing union of Borel equivalence relations $\mathcal{R}_{n}$ each with finite classes of equal size, and with respect to the usual measure on $2^{\mathbb{N}}$, it is p.m.p and ergodic. Given any countable group $\Gamma$, consider the shift action of $\Gamma$ on $2^{\Gamma}$ and let $\mathcal{O}_{\Gamma}$ be the orbit relation determined by the action. Denote by $E_{\Gamma}$ the induced equivalence relation on the free part of the action $F r_{\Gamma}$, namely on the invariant full measure set consisting of points with trivial stabilizer in $\Gamma$. This is again p.m.p, ergodic with respect to the product measure, and in particular it is not measure-theoretically smooth, namely there is no Borel set meeting every class of $E_{\Gamma}$ in a single point. By a special case of the GlimmEffros Dichotomy cf. KM04, Theorem 28.6], there is then a Borel injection $f: 2^{\mathbb{N}} \rightarrow F r_{\Gamma}$ such that $x E_{0} y \Longleftrightarrow f(x) E_{\Gamma} f(y)$. The action of $\Gamma$ being free on $F r_{\Gamma}$, this injection defines a Borel cocycle $\alpha(x, y)$ form $E_{0}$ into $\Gamma$, given by $\alpha(x, y) \cdot f(x)=f(y)$. This cocycle is clearly class injective.

Thus, using the construction described in Lemma 5.1 Theorem 1.2 establishes that orbital entropy and orbital Rokhlin entropy can always be defined for a p.m.p. action of a countable 
group. But furthermore, we have seen above that for essentially free, ergodic actions of $\Gamma$, our notion of orbital Rokhlin entropy coincides with Rokhlin entropy no matter which class-injective cocycle has been chosen, and no matter which bounded hyperfinite exhaustion for the ergodic hyperfinite relation has been used.

5.2. Ergodicity of cocycle extensions. The Shannon-McMillan-Breiman theorem stated in Theorem 1.8, being a pointwise convergence result for orbital entropy of finite partitions, requires an additional ergodicity assumption for its validity, beyond those sufficient to guarantee the existence of orbital entropy itself. We note that an ergodicity assumption also plays a role in the Shannon-McMillan-Breiman theorem for amenable groups, see Li01]. To explain the ergodicity consition in question, let us first recall that in BN15, Def. 2.2] a notion of weak-mixing for a cocycle $\alpha$ on p.m.p. relation $\mathcal{R}$ on $(Y, \nu)$ was defined, as follows. A cocycle $\alpha: \mathcal{R} \rightarrow \Gamma$ is weak-mixing if for every p.m.p. ergodic action of $\Gamma$ on a spaces $(X, \lambda)$, the extended relation $\mathcal{R}^{X}$ on $X \times Y$ is ergodic with respect to the product measure $\lambda \times \nu$. In particular, the relation $\mathcal{R}$ itself must be ergodic. This definition is a natural extension of the notion of weak-mixing for group actions, where the action of $\Gamma$ on a space $(B, \eta)$ (with $\eta$ not necessarily invariant), is called weak-mixing if given any p.m.p. ergodic action of $\Gamma$ on a space $(X, \lambda)$, the product action of $\Gamma$ on $(X \times B, \lambda \times \eta)$ is still ergodic.

Let us now mention some specific classes of groups and their actions, for which explicit information on the ergodicity of the cocycle extension can be provided.

1) Amenable groups. When $\Gamma$ is amenable, referring to the relation $\mathcal{R}$ and the cocycle $\alpha$ defined in $\$ 5.1(1)$, if the p.m.p. $\Gamma$-action on $(Y, \nu)$ is weak-mixing (in the usual sense for group actions), then the $\Gamma$-action on $(X \times Y, \lambda \times \nu)$ is ergodic for every ergodic p.m.p. action of $\Gamma$. Thus, referring to $\$ 5.1(1)$, the cocycle $\alpha: \mathcal{R} \rightarrow \Gamma$ defined there is a weak-mixing cocycle and so here the extended relation $\mathcal{R}^{X}$ is ergodic.

2) Poisson boundaries. When $\Gamma$ is non-amenable, the most important source (but not the only one) of weak-mixing actions of a countable group $\Gamma$ is the set of its actions on Poisson boundaries $B=B(\Gamma, \mu)$ AL05. These actions are amenable as noted above, and satisfy a stronger condition than weak-mixing, namely double ergodicity with coefficients, see $\mathrm{Ka03}$. If $\Gamma$ is a non-amenable group, then the ergodic action on a Poisson boundary $(B, \eta)$ is not measurepreserving, and thus of type $I I I$. The action on the Maharam extension $(B \times \mathbb{R}, \eta \times L)$ is in fact measure-preserving, on a $\sigma$-finite (but not finite) measure space, but the Maharam extension is not necessarily an ergodic action of $\Gamma$. The possibilities for it are determined by the type of the $\Gamma$-action on $(B, \eta)$, and in particular, if it is of type $I I I_{1}$, then the Maharam extension is ergodic. In general, this does not imply that for any ergodic action of $\Gamma$ on $(X, \lambda)$, the Maharam extension of $(X \times B, \lambda \times \eta)$ is ergodic. If indeed this is the case for every p.m.p. action of $\Gamma$, then the action of $\Gamma$ on $(B, \eta)$ is defined in $\mathrm{BN} 13 \mathrm{~b}$ to have stable type $I I I_{1}$.

Assume that the $\Gamma$-action on $(B, \eta)$ is essentially free, and let $(Y, \nu), \mathcal{R}$ and $\alpha$ be as defined $\$ 5.1(2)$. Then the cocycle $\alpha: \mathcal{R} \rightarrow \Gamma$ is injective, defined on a p.m.p. amenable relation, and if the type of the $\Gamma$-action on $(B, \eta)$ is $I I I_{1}$ it is ergodic. If, furthermore, the $\Gamma$-action on $(B, \eta)$ has stable type $I I I_{1}$, then the cocycle $\alpha$ is weak-mixing, and hence $\mathcal{R}^{X}$ is ergodic for every p.m.p. action of $\Gamma$. Thus all the assumptions of the Shannon-McMillan-Breiman theorem are verified in this case, for which examples will be provided below.

3) Non-trivial stable type. If the type of the $\Gamma$-action on $(B, \eta)$ is $I I I_{\tau}$ for some $\tau>0$ then the action of $\Gamma$ on the Maharam extension has a set of ergodic components admitting a free transitive action of the circle group $\mathbb{R} / \mathbb{Z} \cdot \log \tau$. The circle group acts on the Maharam extension and commutes with the $\Gamma$-action. If this is also the situation for the Maharam extension of all the spaces $(X \times B, \lambda \times \eta)$ for every ergodic p.m.p. action of $\Gamma$, then the $\Gamma$-action on $(B, \eta)$ is defined in BN13b to have stable type $\tau$. In that case, it is also possible to prove a version of the ShannonMcMillan-Breiman pointwise convergence theorem, which applies, rather than to the information functions we defined, to a further average of them. In particular, this provides a proof of the Shannon-McMillan mean-convergence theorem in our context. In the interest of brevity, however, we shall provide the details elsewhere. We refer to BN13b] and BN15] for to a detailed discussion 
of type, stable type and Maharam extensions in the context of pointwise ergodic theorems for group actions.

4) Ergodicity and mixing conditions. Given an injective cocycle $\alpha: \mathcal{R} \rightarrow \Gamma$ on an ergodic p.m.p. amenable relation and a p.m.p. action of $\Gamma$ on $(X, \lambda)$, it is possible to develop criteria to show that the extended relation $\mathcal{R}^{X}$ is ergodic, provided that the $\Gamma$-action on $X$ satisfies additional ergodicity or mixing conditions. This implies that the Shannon-McMillan-Breiman theorem is valid for $\Gamma$-actions on a suitable class of p.m.p. actions on $(X, \lambda)$. A simple example of this phenomenon arises for the p.m.p. actions of finitely generated non-abelian free groups $\mathbb{F}_{r}$. Here, taking the boundary $\left(\partial \mathbb{F}_{r}, \nu\right)$ with the uniform measure $\nu$, we construct a cocycle $\alpha: \mathcal{R} \rightarrow \mathbb{F}_{r}$, where $\mathcal{R}$ is an amenable p.m.p. relation on $\partial \mathbb{F}_{r}$, which is not ergodic, but in fact has exactly two ergodic components. If $(X, \lambda)$ is a p.m.p. ergodic action of $\mathbb{F}_{r}$ for which the index 2 subgroup of even length words is ergodic, then the extended relation $\mathcal{R}^{X}$ is an ergodic relation, see BN13a for a detailed exposition. Hence these actions of $\mathbb{F}_{r}$ satisfy the Shannon-McMillan-Breiman theorem. We will give a detailed exposition of this case, which will also demonstrate the geometric significance of the theorem, in the next section.

To conclude this section let us note the following results concerning type and stable type, which are relevant to the foregoing discussion.

Examples 5.2. (1) Let $\Gamma$ be an irreducible lattice in a connected semisimple Lie group with finite center and without compact factors. Then the action of $\Gamma$ on the maximal boundary $(G / P, m)$, where $P$ is a minimal parabolic subgroup and $m$ is the Lebesgue measure class, is amenable and has stable type $I I I_{1}$, see [BN13b].

(2) Let $\Gamma$ be a discrete non-elementary subgroup of isometries of real hyperbolic space. By Su82, Thm 6], the type of the action of $\Gamma$ on the boundary of hyperbolic space with respect to the Lebesgue measure class is $I I I_{1}$, when restricted to the recurrent part. In Sp87 this result was proved for the action of fundamental groups of compact connected negatively curved manifolds acting on the visual boundary with the manifold measure class.

(3) Let $\Gamma$ be a finitely generated free group. By RR97, the action of $\Gamma$ has non-trivial type with respect to harmonic measures, namely stationary measures of suitable random walks. For $\Gamma$ being a word hyperbolic group, it was proved in INO08 that the action of $\Gamma$ on the Poisson boundary associated with a generating measure of finite support has non-trivial type.

(4) In Bo14 it was proved that for word hyperbolic groups (with an additional technical condition) the action on the Gromov boundary with respect to a quasi-conformal measure (and in particular the Patterson-Sullivan measure) measure has non-trivial stable type.

5.3. Ergodicity of the extended relation for strongly mixing actions. We now turn to show that in fact the Shannon-McMillan-Breiman theorem holds for all free strongly mixing actions of all countable groups, provided the equivalence relation $\mathcal{R}$ and the cocycle $\alpha$ are chosen suitably. We have seen in Theorem [1.8 that it suffices to ensure that the extended relation is ergodic. Choosing $\mathcal{R}$ and $\alpha$ suitably, this will indeed be the case, and this fact relies on the following result, communicated to us by A. Kechris.

Proposition 5.3. Ke17 For every p.m.p. free action $(X, \mu)$ of a countable group $\Gamma$ which is strongly mixing, there exists a p.m.p. ergodic hyperfinite relation $\mathcal{R}$ and a class injective cocycle $\alpha: \mathcal{R} \rightarrow \Gamma$, such that the extended relation $\mathcal{R}^{X}$ is ergodic.

Proof. Consider any ergodic p.m.p. action $(X, \mu)$ of any countable group $\Gamma$, and the associated equivalence relation $\mathcal{O}_{\Gamma}$ determined by the orbits of $\Gamma$ in $X$. It is well known (see e.g. Ke10, Theorem 3.5]) that $\mathcal{O}_{\Gamma}$ contains as a subrelation the orbit relation of a free measure-preserving action of a suitable element $T$ in the full group. Since $T$ is orbit equivalent to a free ergodic action of the group $\bigoplus_{\mathbb{Z}} \mathbb{Z}_{2}$ (e.g. by CFW81), it follows that $\mathcal{O}_{\Gamma}$ also contains an ergodic hyperfinite p.m.p. relation $\mathcal{R}$. Clearly, if the $\Gamma$-action on $X$ is free, then there is a class injective cocycle $\alpha: \mathcal{R} \rightarrow \Gamma$, given by $\alpha(\gamma x, x)=\gamma$, whenever $(\gamma x, x) \in \mathcal{R}$. As already noted, by CFW81 the relation $\mathcal{R}$ is generated by a p.m.p. free action of $\mathbb{Z}$, and therefore using Dye's theorem Dy59 on 
the orbit equivalence of any two free p.m.p. actions of $\mathbb{Z}$, we can assume without loss of generality that $\mathcal{R}$ is generated by a strongly mixing free p.m.p. action of $\mathbb{Z}$ on $(X, \mu)$. Thus there exists a strongly mixing p.m.p. map $T: X \rightarrow X$ whose orbits in $X$ are precisely the equivalence classes of $\mathcal{R}$. The extended relation $\mathcal{R}^{X}$ on $X \times X$ using the cocycle $\alpha$ that we have used throughout is then also generated by a p.m.p. action of $\mathbb{Z}$ on $X \times X$, given by $T_{X}\left(x, x^{\prime}\right)=\left(T x, \alpha(T x, x) x^{\prime}\right)$. Now note that if the original $\Gamma$-action on $(X, \mu)$ is strongly mixing, namely the matrix coefficients vanish at infinity in $\Gamma$, then the extended action of $\mathbb{Z}$ on $X \times X$ given by the action of $T_{X}$ must be strongly mixing as well. It suffices to check this fact on a dense subspace of functions in $L^{2}(X \times X)$, and we choose this dense subspace to consist of product functions $f(x) f^{\prime}\left(x^{\prime}\right)$, with $f, f^{\prime} \in L^{2}(X) \cap L^{\infty}(X)$. Then

$$
\begin{gathered}
\left\langle\left(T_{X}\right)^{n}\left(f f^{\prime}\right), g g^{\prime}\right\rangle=\int_{X \times X} f\left(T^{n} x\right) f^{\prime}\left(\alpha\left(T^{n} x, x\right) x^{\prime}\right) g(x) g^{\prime}\left(x^{\prime}\right) d \lambda(x) d \lambda\left(x^{\prime}\right) \\
=\int_{X} f\left(T^{n} x\right) g(x)\left(\int_{X} f^{\prime}\left(\alpha\left(T^{n} x, x\right) x^{\prime}\right) g^{\prime}\left(x^{\prime}\right) d \lambda\left(x^{\prime}\right)\right) d \lambda(x)
\end{gathered}
$$

Note that for any given $T$-orbit in $X$, namely for each given class of $\mathcal{R}$, the cocycle $\alpha$ is class injective, and hence the values of the cocycle $\alpha$ on that class must converge to infinity in $\Gamma$. It follows for almost any $x \in X, \alpha\left(T^{n} x, x\right)$ converges to infinity in $\Gamma$. By strong mixing of $\Gamma$ on $X$ the inner integral converges to $\int_{X} f^{\prime} g^{\prime} d \lambda\left(x^{\prime}\right)$, and by Lebesegue dominated convergence theorem, the doubly integral then converges to $\int_{X \times X} f f^{\prime} \cdot \int_{X} g g^{\prime}$, so that $T_{X}$ is strongly mixing.

Finally, having shown that $T_{X}$ acting on $X \times X$ is strongly mixing, we can certainly conclude that it is ergodic, and it follows that the equivalence relation determined by the $T_{X}$-orbits in $X \times X$ is an ergodic relation. By construction this relation is precisely the extended relation associated $\mathcal{R}^{X}$ associated with the cocycle $\alpha$, so that the extended relation is indeed ergodic.

5.4. Ergodicity of the extended relation via weakly mixing Bernoulli actions. In a recent paper VW18, Vaes and Wahl show that there is a very large collection of countable groups $\Gamma$ which admit a nonsingular Bernoulli action of type $I I I_{1}$. They verify this for instance for groups containing at least one element of infinite order and conjecture that $\Gamma$ admits a nonsingular Bernoulli action $\Gamma \curvearrowright(B, \eta)$ of type $I I I_{1}$ if and only if the first $L^{2}$-cohomology of $\Gamma$ vanishes. These Bernoulli actions give rise to essentially free, p.m.p. and weakly mixing Maharam extensions, say $\Gamma \curvearrowright\left(B \times \mathbb{R}, \eta \times e^{t} d t\right)$, see [VW18, Theorems 5.1 and 6.1]. The weak mixing condition implies that for any p.m.p. ergodic action of $\Gamma$ on $(X, \mu)$, the action of $\Gamma$ on $\left(X \times B \times \mathbb{R}, \mu \times \eta \times e^{t} d t\right)$ is ergodic. Now restrict the orbit relation of $\Gamma$ in this action to the probability space $X \times Y$, where $Y:=B \times(-\infty, 0)$ and $\nu:=\eta \times e^{t} d t$, which we denote by $\mathcal{S}=\mathcal{S}(X, B)$.

As we saw in the proof of Lemma 5.1 the relation $\mathcal{S}$ admits an ergodic, hyperfinite subequivalence relation $\mathcal{S}_{0}=\mathcal{S}_{0}(X, B)$. By freeness of the original $\Gamma$-action on $X$, we obtain a canonical cocycle defined over the hyperfinite p.m.p. ergodic equivalence relation $\mathcal{S}_{0}$, given by $\alpha: \mathcal{S}_{0} \rightarrow \Gamma$, where $\alpha\left((x, y),\left(x^{\prime}, y^{\prime}\right)\right)=g$ for the unique element $g \in \Gamma$ which satisfies $g \cdot\left(x^{\prime}, y^{\prime}\right)=(x, y)$. This way, the ergodicity assumption required for the validity of the convergence result appearing in the Shannon-McMillan-Breiman theorem, cf. Theorem 1.8, is guaranteed to hold. Note however, that the present set-up is different than the set-up considered previously, since the relation $\mathcal{S}_{0}$ and the cocycle $\alpha$ depend on $X$ and $B$. Here we do not establish ergodicity of the extended relation via properties of one fixed auxiliary equivalence relation $\mathcal{R}$ and cocycle $\alpha$. Rather, we obtain the ergodicity of $\mathcal{S}_{0}$ directly, as an equivalence relation on $(X \times Y, \mu \times \nu)$, using weak-mixing of $B \times \mathbb{R}$. This argument, unlike our previous one, cannot be used in conjunction with the subadditive principle, and therefore the limit in the convergence result that it provides is not shown to be independent of the cocycle $\alpha$ and the space $B$.

\section{THE FREE GROUP}

6.1. The boundary of the free group. We briefly describe the ingredients we need for our analysis, following the exposition of BN13a.

Let $\mathbb{F}=\left\langle a_{1}, \ldots, a_{r}\right\rangle$ be the free group of rank $r \geq 2$, with $S=\left\{a_{i}^{ \pm 1}: 1 \leq i \leq r\right\}$ a free set of generators. The (unique) reduced form of an element $g \in \mathbb{F}$ is the expression $g=s_{1} \cdots s_{n}$ with 
$s_{i} \in S$ and $s_{i+1} \neq s_{i}^{-1}$ for all $i$. Define $|g|:=n$, the length of the reduced form of $g$. The distance function on $\mathbb{F}$ is defined by $d\left(g_{1}, g_{2}\right):=\left|g_{1}^{-1} g_{2}\right|$. The Cayley graph associated with the generating set $S$ is a regular tree of valency $2 r$, and $d$ coincides with its edge-path distance.

The boundary of $\mathbb{F}$ is the set of all sequences $\xi=\left(\xi_{1}, \xi_{2}, \ldots\right) \in S^{\mathbb{N}}$ such that $\xi_{i+1} \neq \xi_{i}^{-1}$ for all $i \geq 1$. We denote it by $\partial \mathbb{F}$. A metric $d_{\partial}$ on $\partial \mathbb{F}$ is defined by $d_{\partial}\left(\left(\xi_{1}, \xi_{2}, \ldots\right),\left(t_{1}, t_{2}, \ldots\right)\right)=\frac{1}{n}$ where $n$ is the largest natural number such that $\xi_{i}=t_{i}$ for all $i<n$. If $\left\{g_{i}\right\}_{i=1}^{\infty}$ is any sequence of elements in $\mathbb{F}$ and $g_{i}:=t_{i, 1} \cdots t_{i, n_{i}}$ is the reduced form of $g_{i}$ then $\lim _{i} g_{i}=\left(\xi_{1}, \xi_{2}, \ldots\right) \in \partial \mathbb{F}$ if $t_{i, j}$ is eventually equal to $\xi_{j}$ for all $j$. If $\xi \in \partial \mathbb{F}$ then we will write $\xi_{i} \in S$ for $i$-th element in the sequence $\xi=\left(\xi_{1}, \xi_{2}, \xi_{3}, \ldots\right)$.

We define a probability measure $\nu$ on $\partial \mathbb{F}$, by the requirement that every finite sequence $t_{1}, \ldots, t_{n}$ with $t_{i+1} \neq t_{i}^{-1}$ for $1 \leq i<n$, the following holds :

$$
\nu\left(\left\{\left(\xi_{1}, \xi_{2}, \ldots\right) \in \partial \mathbb{F}: \xi_{i}=t_{i} 1 \leq i \leq n\right\}\right):=\left|S_{n}(e)\right|^{-1}=(2 r-1)^{-n+1}(2 r)^{-1} .
$$

There is a natural action of $\mathbb{F}$ on $\partial \mathbb{F}$ by

$$
\left(t_{1} \cdots t_{n}\right) \xi:=\left(t_{1}, \ldots, t_{n-k}, \xi_{k+1}, \xi_{k+2}, \ldots\right)
$$

where $t_{1}, \ldots, t_{n} \in S, t_{1} \cdots t_{n}$ is in reduced form and $k$ is the largest number $\leq n$ such that $\xi_{i}^{-1}=t_{n+1-i}$ for all $i \leq k$. Observe that if $g=t_{1} \cdots t_{n}$ then the Radon-Nikodym derivative satisfies

$$
\frac{d \nu \circ g}{d \nu}(\xi)=(2 r-1)^{2 k-n}
$$

6.2. The horospherical relation and the fundamental cocycle. Let $\mathcal{R}$ be the equivalence relation on $\partial \mathbb{F}$ given by $(\xi, \eta) \in \mathcal{R}$ if and only when writing $\xi=\left(\xi_{1}, \xi_{2}, \ldots\right)$ and $\eta=\left(\eta_{1}, \eta_{2}, \ldots\right)$, there exists $n$ such that $\eta_{i}=\xi_{i}$ for all $i>n$. Thus $\eta \mathcal{R} \xi$ if and only if $\eta$ and $\xi$ have the same synchronous tail, if and only if they differ by finitely many coordinates only.

Let $\mathcal{R}_{n}$ be the equivalence relation given by $(\xi, \eta) \in \mathcal{R}_{n}$ if and only if $\xi_{i}=\eta_{i} \forall i>n$. Then $\mathcal{R}$ is the increasing union of the finite subequivalence relations $\mathcal{R}_{n}$. Thus $\mathcal{R}$ is a hyperfinite relation.

Consider the relation $\mathcal{R}^{\prime}$ on $\partial \mathbb{F}$ such that $\eta \mathcal{R}^{\prime} \xi$ if and only if there is a $g \in \mathbb{F}$ such that $g \xi=\eta$ and $\frac{d \nu \circ g}{d \nu}(\xi)=1$. Note that the level set of the Radon-Nikodym derivative, namely $\left\{g \in \mathbb{F} ; \frac{d \nu \circ g}{d \nu}(\xi)=1\right\}$ is the horosphere in the Cayley tree based at $\xi$ and passing through the identity in $\mathbb{F}$. Note that in that case $\xi=g^{-1} \eta$, and $\frac{d \nu \circ g^{-1}}{d \nu}(\eta)=\left(\frac{d \nu \circ g}{d \nu}(\xi)\right)^{-1}=1$, so that the relation is indeed symmetric. The transitivity of the horospherical relation $\mathcal{R}^{\prime}$ follows from the cocycle identity which the Radon-Nikodym derivative satisfies. Thus $\mathcal{R}^{\prime}$ is an equivalence relation, and by definition, the measure $\nu$ is $\mathcal{R}^{\prime}$-invariant.

The relation $\mathcal{R}^{\prime}$ on $\partial \mathbb{F}_{r}$ can also be defined more concretely by the condition that $(\xi, \eta) \in \mathcal{R}^{\prime}$ iff there exists $k$ s.t. $\eta=g \xi$ and $g=\eta_{1} \cdot \ldots \cdot \eta_{k} \cdot \xi_{k}^{-1} \cdot \ldots \cdot \xi_{1}^{-1}$. It follows that $\eta=g \xi$ has the same synchronous tail as $\xi$ from the $k+1$-th letter onwards. Equivalently, $g^{-1}$ belongs to the horosphere based at $\xi$ and passing through the identity in $\mathbb{F}_{r}$, namely the geodesic from $g^{-1}$ to $\xi$ and the geodesic from $e$ to $\xi$ meet at a point which is equidistant from $e$ and $g^{-1}$. Thus it is natural to call $\mathcal{R}^{\prime}$ the horospherical relation and the equivalence class of $\xi$ under $\mathcal{R}_{n}$ the horospherical ball of radius $n$ based at $\xi$. Since $\xi$ and $\eta=g \xi$ have the same synchronous tail, $\mathcal{R}^{\prime}$ coincides with the synchronous tail relation $\mathcal{R}$.

The fundamental cocycle of the tail relation is the measurable map $\alpha: \mathcal{R} \rightarrow \mathbb{F}_{r}$ given, for $\eta=\left(\eta_{1}, \ldots, \eta_{k}, \ldots\right)$ and $\xi=\left(\xi_{1}, \ldots, \xi_{k}, \ldots\right)\left(\right.$ with $\left.(\eta, \xi) \in \mathcal{R}_{k}\right)$, by

$$
\alpha(\eta, \xi)=\eta_{1} \cdot \ldots \cdot \eta_{k} \cdot \xi_{k}^{-1} \cdot \ldots \cdot \xi_{1}^{-1}
$$

so that $\alpha(\eta, \xi) \xi=\eta$. The cocycle takes values in the subgroup $\mathbb{F}_{r}^{e}$ consisting of words of even length, and more precisely for each $k$ the set of values $\alpha(\eta, \xi)$ for fixed $\eta$ and $\xi \in \mathcal{R}_{k}(\eta)$ coincides with the intersection of the word metric ball $B_{2 k}(e)$ with the horoball based at $\eta$ and passing through $e$. This set is called the horospherical ball of radius $2 k$ determined by $\eta$ and denoted by $B_{2 k}^{\eta}$. 
Note further that the set of cocycle values $\alpha(\xi, \eta)$ for $\xi \mathcal{R}_{k} \eta$ with $\eta$ fixed, namely the set $\left(B_{2 k}^{\eta}\right)^{-1}$, is given by

$$
\alpha(\xi, \eta)=\xi_{1} \cdot \ldots \cdot \xi_{k} \cdot \eta_{k}^{-1} \cdot \ldots \cdot \eta_{1}^{-1}
$$

and this set contains words of length at most $2 k$ whose first $(k-1)$ letters can be specified arbitrarily.

Finally, consider the set of values of the cocycles $\alpha(\eta, \xi)$ for all points $\eta \neq \xi$ which are $\mathcal{R}_{k}$ equivalent to another, i.e. as we go over all pairs of distinct points in some equivalence class of the form $\mathcal{R}_{k}(\zeta)$. This set of values clearly contains all the even words in a ball of radius $2 k-2$, i.e. $B_{2 k-2}(e) \cap \mathbb{F}_{r}^{e}$.

The finite order automorphisms of $\mathcal{R}$ is the subgroup $\Phi$ of $\cup_{n \in \mathbb{N}}\left[\mathcal{R}_{n}\right]$ generated by the transformations defined as follows. Let $\pi_{n}: \partial \mathbb{F}_{r} \rightarrow S^{n}$ be the projection given by $\pi_{n}\left(s_{1}, s_{2}, \ldots\right)=$ $\left(s_{1}, s_{2}, \ldots, s_{n}\right)$. We say that a map $\psi: \partial \mathbb{F}_{r} \rightarrow \partial \mathbb{F}_{r}$ has order $n$ if $\psi(\xi)=\psi\left(\xi^{\prime}\right)$ for any two boundary points $\xi, \xi^{\prime} \in \partial \mathbb{F}_{r}$ with $\pi_{n}(\xi)=\pi_{n}\left(\xi^{\prime}\right)$.

For any $\left(\xi, \xi^{\prime}\right) \in \mathcal{R}$ there exists a map $\phi \in \Phi$ such that $\phi(\xi)=\xi^{\prime}$ and $\phi$ has order $n$ for some $n \in \mathbb{N}$, see BN13a]. Thus the group of finite order automorphisms clearly generates the synchronous tail (i.e. the horospherical) relation.

The extended horospherical relation. Let $\mathbb{F}$ act by measure-preserving transformations on a probability space $(X, \lambda)$. Let $\mathcal{R}_{n}^{X}$ be the equivalence relation on $X \times \partial \mathbb{F}$ defined by $\left((x, \xi),\left(x^{\prime}, \xi^{\prime}\right)\right) \in$ $\mathcal{R}_{n}^{X}$ if and only if there exists $g \in \mathbb{F}$ with $(g x, g \xi)=\left(x^{\prime}, \xi^{\prime}\right)$ and $\left(\xi, \xi^{\prime}\right) \in \mathcal{R}_{n}$ (i.e., if $\xi=\left(\xi_{1}, \ldots\right) \in$ $S^{\mathbb{N}}$ and $\xi^{\prime}=\left(\xi_{1}^{\prime}, \ldots\right) \in S^{\mathbb{N}}$ then $\xi_{i}=\xi_{i}^{\prime}$ for all $\left.i \geq n\right)$.

Inspecting the definitions, we see that the extended horospherical relation on $X \times \partial \mathbb{F}_{r}$ coincides with the extension of the horospherical (i.e. synchronous tail) relation $\mathcal{R}$ on $\partial \mathbb{F}_{r}$ via the fundamental cocycle $\alpha: \mathcal{R} \rightarrow \mathbb{F}_{r}$ defined above.

It is easy to see that for any $(y, \xi) \in X \times \partial \mathbb{F}$,

$$
\left.\mid \mathcal{R}_{n}^{X}(y, \xi)\right)|=| \mathcal{R}_{n}(\xi) \mid=(2 r-1)^{n} .
$$

The relation $\mathcal{R}^{X}=\bigcup_{n>1} \mathcal{R}_{n}^{X}$ is thus a hyperfinite measurable equivalence relation, it preserves the measure $\nu \times \lambda$, and it is uniform, namely for each $n \geq 1$ almost every equivalence class of the relation $\mathcal{R}_{n}^{X}$ has the same cardinality.

6.3. Shannon-McMillan-Breiman theorem for the free groups. Let $\mathcal{R}^{X}$ be the equivalence relation on $X \times \partial \mathbb{F}_{r}$. We may assume the action of $\mathbb{F}_{r}$ on $(X, \lambda)$ is ergodic, and we will use the following.

Theorem 6.1. BN13a. If $\mathbb{F}_{r}^{e}$ acts on $(X, \lambda)$ ergodically, then the diagonal action $\mathbb{F}_{r}^{e} \curvearrowright X \times \partial \mathbb{F}_{r}$ is ergodic.

The type of the boundary action. The type of the action $\mathbb{F}_{r} \curvearrowright\left(\partial \mathbb{F}_{r}, \nu\right)$ is $I I I_{\tau}$ where $\tau=(2 r-1)^{-1}$. It is follows from BN13a, Theorem 4.1] that the stable type of $\mathbb{F}_{r} \curvearrowright\left(\partial \mathbb{F}_{r}, \nu\right)$ is $I I I_{\tau^{2}}$. In fact, if $\mathbb{F}_{r}^{e}$ denotes the index 2 subgroup of $\mathbb{F}$ consisting of all elements of even word length then $\mathbb{F}_{r}^{e} \curvearrowright\left(\partial \mathbb{F}_{r}, \nu\right)$ is of type $I I I_{\tau^{2}}$ and stable type $I I I_{\tau^{2}}$. It is also weakly mixing. Indeed, $\left(\partial \mathbb{F}_{r}, \nu\right)$ is naturally identified with $B\left(\mathbb{F}_{r}, \mu\right)$, the Poisson boundary of the random walk generated by the measure $\mu$ that is distributed uniformly on $S$. By AL05, the action of any countable group $\Gamma$ on the Poisson boundary $B(\Gamma, \kappa)$ is weakly mixing whenever the measure $\kappa$ is adapted. This shows that $\mathbb{F}_{r} \curvearrowright\left(\partial \mathbb{F}_{r}, \nu\right)$ is weakly mixing. Moreover, if we denote $S^{2}=\{s t: s, t \in S\}$, then $\left(\partial \mathbb{F}_{r}, \nu\right)$ is naturally identified with the Poisson boundary $B\left(\mathbb{F}_{r}^{e}, \mu_{2}\right)$ where $\mu_{2}$ is the uniform probability measure on $S^{2}$. So the action $\mathbb{F}_{r}^{e} \curvearrowright(\partial \mathbb{F}, \nu)$ is also weakly mixing.

Let $\mathbb{F}_{r}$ act on $\partial \mathbb{F}_{r} \times \mathbb{Z}$ by $g(b, t)=\left(g b, t-\log r_{\nu}(g, b)\right)$, which gives the discrete Maharam extension in this case. Let $\mathcal{R}$ be the orbit-equivalence relation restricted to $\partial \mathbb{F}_{r} \times\{0\}$, which we may, for convenience, identify with $\partial \mathbb{F}_{r}$. In other words, $b \mathcal{R} b^{\prime}$ if and only if there is an element $g \in \mathbb{F}_{r}$ such that $g b=b^{\prime}$ and $\frac{d \nu \circ g}{d \nu}(b)=1$. As noted above, this is the same as the (synchronous) tail-equivalence relation on $\mathbb{F}_{r}$. In other words, two elements $\xi=\left(\xi_{1}, \xi_{2}, \ldots\right), \eta=\left(\eta_{1}, \eta_{2}, \ldots\right) \in \partial \mathbb{F}_{r}$ are $\mathcal{R}$-equivalent if and only if there is an $m$ such that $\xi_{n}=\eta_{n}$ for all $n \geq m$. But now note that if $b \mathcal{R}(g b)$, then necessarily $g \in \mathbb{F}_{r}^{e}$. So $\mathcal{R}$ can also be $\mathrm{r}$ 
Let $\alpha: \mathcal{R} \rightarrow \mathbb{F}_{r}^{e}$ be the cocycle $\alpha(g b, b)=g$ for $g \in \mathbb{F}_{r}^{e}, b \in \partial \mathbb{F}_{r}$. This is well-defined almost everywhere because the action of $\mathbb{F}_{r}^{e}$ is essentially free. As noted above, given two $\mathcal{R}_{k}$-equivalent points $\xi, \eta \in \partial \mathbb{F}_{r}$ as above, the cocycle value is given by $\alpha(\eta, \xi)=\eta_{1} \cdot \ldots \cdot \eta_{k} \cdot \xi_{k}^{-1} \cdot \ldots \cdot \xi_{1}^{-1}$.

Because $\mathbb{F}_{r}^{e} \curvearrowright\left(\partial \mathbb{F}_{r}, \nu\right)$ has type $I I I_{\tau^{2}}$ and stable type $I I I_{\tau^{2}}$, this cocycle is weakly mixing for $\mathbb{F}_{r}^{e}$. In other words, if $\mathbb{F}_{r}^{e} \curvearrowright(X, \mu)$ is any ergodic p.m.p action, then the equivalence relation $\mathcal{R}^{X}$ defined on $X \times \partial \mathbb{F}_{r}$ by the cocycle extension is ergodic. Since the relation is hyperfinite and the cocycle is injective, we conclude that the action of $\mathbb{F}_{r}$ on any ergodic p.m.p. space satisfies the Shannon-McMillan-Breiman theorem, provided only that $\mathbb{F}_{r}^{e}$ acts ergodically on $X$. Applying Theorem 1.8, we conclude :

Theorem 6.2. Let $(X, \mu)$ be any essentially free p.m.p. action of the free group $\mathbb{F}_{r}, r \geq 2$. Assume that the action is ergodic under the index 2 subgroup of even words $\mathbb{F}_{r}^{e}$. Fix any finite partition $\mathcal{P}$ of $X$. For a boundary point $\eta \in \partial \mathbb{F}_{r}$, consider the sequence of partitions obtained from $\mathcal{P}$ by refining it along the horospherical balls $B_{2 k}^{\eta}$ determined by $\eta$ :

$$
\bigvee_{g \in B_{2 k}^{\eta}} g \mathcal{P}=\bigvee_{\xi \in \mathcal{R}_{k}(\eta)} \alpha(\xi, \eta)^{-1} \mathcal{P}
$$

Then the information functions of the refined partitions converge to the orbital entropy (of the partition $\mathcal{P}$ ) for the action of $\mathbb{F}_{r}$ on $X$, for $\mu$-almost every $x \in X$, for $\nu$-almost every $\eta \in \mathbb{F}_{r}$.

\section{REFERENCES}

[AL05] Aaronson, J. and Lemanczyk, M. Exactness of Rokhlin endomorphisms and weak mixing of Poisson boundaries. Algebraic and topological dynamics. Contemp. Math. 385, Amer. Math. Soc., Providence, RI (2005), pp. 77-87.

[Br57] Breiman, L., The individual ergodic theorem of information theory, Ann. Math. Stat. 28 (1957), pp. 809-911. Correction, ibid. 31 (1960), pp. 809-910.

[Bo10] Bowen, L. Measure conjugacy invariants for actions of countable sofic groups. J. Amer. Math. Soc. 23 (2010), pp. 217-245.

[Bo12] Bowen, L. Sofic entropy and amenable groups. Ergodic Theory Dynam. systems 32, no. 2 (2012), pp. $427-466$.

[Bo14] Bowen, L. The type and stable type of the boundary of a Gromov hyperbolic group. Geometriae Dedicata 172 (2014), pp. 363-386.

[BN13a] Bowen, L. and Nevo, A. Geometric covering arguments and ergodic theorems for free groups. L'Enseignement Mathématique, 59 (2013), pp. 133-164.

[BN13b] Bowen, L. and Nevo, A. Pointwise ergodic theorems beyond amenable groups. Ergod. Th. and Dynam. Sys. 33 (2013), pp. 777-820.

[BN15] Bowen, L. and Nevo, A. Amenable equivalence relations and the construction of ergodic averages for group actions. To appear in Journal d'Analyse Mathématique,(2015).

[CFW81] Connes, A., Feldman, J. and Weiss, B. An amenable equivalence relation is generated by a single transformation. Ergod. Th. and Dynam. Sys. 1 (1981), pp. 431-450.

[Da01] Danilenko, A., Entropy Theory from the Orbital Point of View. Monatsh. Math. 134 (2001), pp. 121-141.

[DP02] Danilenko, A. and Park, K. Generators and Bernoullian factors for amenable actions and cocycles on their orbits. Ergod. Th. and Dynam. Sys. (2002) 22, pp. 1715-1745.

[Dy59] Dye, H. On groups of measure transformations I. Amer. J. Math., vol. 81 (1959), pp. 119-159.

[FM77] Feldman, J. and Moore, C. C. Ergodic equivalence relations, von Neumann algebras and cohomology. I and II. Trans. A.M.S. 234 (1977), pp. 289-324

[FS08] Flajolet, P. and Sedgewick, R. Analytic Combinatorics. Cambridge University Press New York, 2008.

[Ga00] Gaboriau, D. On orbit equivalence of measure preserving actions. Rigidity in dynamics and geometry (Cambridge, 2000), Springer Berlin 2002, pp. 167-186.

[INO08] Izumi, M., Neshveyev, S. and Okayasu, R. The ratio set of the harmonic measure of a random walk on a hyperbolic group. Israel J. Math. 163 (2008), pp. 285-316.

[Ka03] Kaimanovich, V. A. Double ergodicity of the Poisson boundary and applications to bounded cohomology. Geom. Funct. Anal. 13 (2003), no. 4, pp. 852-861.

[Ke10] Kechris, A. Global Aspects of Ergodic Group Actions. Math. Surveys and Monographs 160. Amer. Math. Soc. Providence RI, 2010.

[Ke17] A. Kechris, personal communication, Caltech, May 2017.

[KM04] A. Kechris and B. Miller, Topics in orbit equivalence. Lecture Notes in Mathematics 1852, Springer Berlin Heidelberg New York, 2004.

[Ke13] Kerr, D. Sofic measure entropy via finite partitions. Groups. Geom. Dyn. 7 (2013), pp. 617-632.

[KL11] Kerr, D. and Li, H. Entropy and the variational principle for actions of sofic groups. Invent. Math., 186 (2011), pp. 501-558. 
[KL13] Kerr, D. and Li, H. Soficity, amenability, and dynamical entropy. Amer. J. Math. 135 (2013), pp. 721-761.

[Ki75] Kieffer, J. C., A ratio limit theorem for a strongly subadditive set function in a locally compact amenable group. Pacific Journal of Mathematics 61 (1975), pp. 183-190.

[Ko58] Kolmogorov, A.N. New Metric Invariant of Transitive Dynamical Systems and Endomorphisms of Lebesgue Spaces. (Russian) Doklady of Russian Academy of Sciences 119 (1958), pp. 861-864.

[Ko59] Kolmogorov, A.N. Entropy per unit time as a metric invariant for automorphisms (Russian) Doklady of Russian Academy of Sciences 124 (1959), pp. 754-755.

[Li01] Lindenstrauss, E. Pointwise theorems for amenable groups. Invent. Math., 146 (2001) pp. $259-295$.

[Mc53] McMillan, B., The basic theorems of information theory. Ann. Math. Stat. 24 (1953), pp. $196-219$.

[Ol85] Ollagnier, J., Ergodic Theory and Statistical Mechanics. Lecture Notes in Mathematics 1115, SpringerVerlag, Berlin, 1985.

[OW80] Ornstein, D. and Weiss, B. Ergodic theory of amenable group actions. I. The Rohlin lemma., Bull. Amer. Math. Soc. vol. 2 (1980), pp. 161-164.

[OW83] Ornstein, D. and Weiss, B. The Shannon-McMillan-Breiman theorem for a class of amenable groups. Israel J. Math. 44 (1983) pp. 53-60.

[OW87] Ornstein, D. and Weiss, B. Entropy and isomorphism theorems for actions of amenable groups. Journal d'Analyse Mathématique 48 (1987), pp. 1-141.

[RR97] Ramagge, J. and Robertson, G. Factors from trees. Proc. Amer. Math. Soc. 125 no. 7 (1997), pp. 2051-2055.

[Se14] Seward, B. Krieger's finite generator theorem for ergodic actions of countable groups I, to appear in Invent. Math., http://arxiv.org/abs/1405.3604 (2014).

[Se15b] Seward, B. Krieger's finite generator theorem for ergodic actions of countable groups II, to appear in J. Modern Dynamics. http://arxiv.org/abs/1501.03367 (2015).

[Se16] Seward, B. Weak containment and Rokhlin entropy. http://arxiv.org/abs/1602.06680 (2016).

[Sh48] Shannon, C. E., A mathematical theory of communication. Bell System Tech. J. 27 (1948), pp. 379-423, pp. 623-656.

[Si59] Sinai, Y. On the concept of entropy for a dynamical system. Dokl. Akad. Nauk SSSR 124 (1959), pp. $768-771$.

[Sp87] Spatzier, R. J. An example of an amenable action from geometry. Ergodic Theory Dynam. Systems 7 no. 2 (1987), pp. 289-293.

[ST12] Seward, B. and Tucker-Drob, R. Borel structurability on the 2-shift of a countable group. Ann. Pure and Applied Logic 167 (2016).

[Su82] Sullivan, D. Discrete conformal groups and measurable dynamics. Bull. Amer. Math. Soc. (N.S.) 6 no. 1 (1982), pp. 57-73.

[VW18] Vaes, S. and Wahl, J., Bernoulli actions of type $I I I_{1}$ and $L^{2}$-cohomology. Geom. Func. Ana. 28, 518-562, (2018).

[We84] Weiss, B. Measurable dynamics. Contemp. Math. vol. 26 (1984), pp. 395-421.

[We03] Weiss, B. Actions of amenable groups. London Mathematical Society Lecture Note Series 310, 226-262, (2003).

[Zi78] Zimmer, R. Amenable ergodic group actions and an application to Poisson boundaries of random walks. J. Funct. Anal. 27 (1978), 350-372.

Technion, IsRael Institute of Technology

E-mail address: anevo@tx.technion.ac.il

UNIVERSITY OF LEIPZIG

E-mail address: felix.pogorzelski@math.uni-leipzig.de 